\title{
Cholinergic Control in Developing Prefrontal-Hippocampal Networks
}

\author{
P. Christoph Janiesch, Hanna-Sophie Krüger, Beatrice Pöschel, and Ileana L. Hanganu-Opatz \\ Developmental Neurophysiology, Center for Molecular Neurobiology, University Medical Center Hamburg-Eppendorf, 20251 Hamburg, Germany
}

The cholinergic drive enhances input processing in attentional and mnemonic context by interacting with the activity of prefrontalhippocampal networks. During development, acetylcholine modulates neuronal proliferation, differentiation, and synaptic plasticity, yet its contribution to the maturation of cognitive processing resulting from early entrainment of neuronal networks in oscillatory rhythms remains widely unknown. Here we show that cholinergic projections growing into the rat prefrontal cortex (PFC) toward the end of the first postnatal week boost the generation of nested gamma oscillations superimposed on discontinuous spindle bursts by acting on functional muscarinic but not nicotinic receptors. Although electrical stimulation of cholinergic nuclei increased the occurrence of nested gamma spindle bursts by $41 \%$, diminishment of the cholinergic input by either blockade of the receptors or chronic immunotoxic lesion had the opposite effect. This activation of locally generated gamma episodes by direct cholinergic projections to the PFC was accompanied by indirect modulation of underlying spindle bursts via cholinergic control of hippocampal theta activity. With ongoing maturation and switch of network activity from discontinuous bursts to continuous theta-gamma rhythms, accumulating cholinergic projections acting on both muscarinic and nicotinic receptors mediated the transition from high-amplitude slow to low-amplitude fast rhythms in the PFC. By exerting multiple actions on the oscillatory entrainment of developing prefrontal-hippocampal networks, the cholinergic input may refine them for later gating processing in executive and mnemonic tasks.

\section{Introduction}

Acetylcholine (ACh) controls the content and the intensity of conscious awareness. Initiated by the pioneering work of MacIntosh and Oboring (1955), which demonstrated that ACh controls the neuronal excitability, the interest in the function of the cholinergic system greatly increased with the characterization of cholinergic antagonists as impairment agents of cognitive abilities (Deutsch, 1971; Drachman, 1977) and with the postmortem identification of reduced cholinergic markers in the brain of Alzheimer's disease patients (Bowen et al., 1976; Perry et al., 1978). The cholinergic drive controls a large variety of cognitive processes, such as attention (Hasselmo and Sarter, 2011), learning (Fine et al., 1997; Miranda and Bermúdez-Rattoni, 1999), and memory (Hasselmo and Bower, 1992; Gil-Bea et al., 2010) by boosting the signal-to-noise ratio (Sillito and Kemp, 1983) in various cortical and subcortical nuclei entrained within a complex neuronal network (Everitt and Robbins, 1997). The mutually interacting PFC and hippocampus (Hipp) represent the core of this network involved in cognitive processing (GoldmanRakic, 1995; Thierry et al., 2000; Warburton and Brown, 2010).

Received May 27, 2011; revised 0ct. 6, 2011; accepted 0ct. 12, 2011.

Author contributions: I.L.H.-0. designed research; P.C.J., H.-S.K., B.P., and I.L.H.-0. performed research; P.C.J., H.-S.K., B.P., and I.L.H.-O. analyzed data; I.L.H.-O. wrote the paper.

I.L.H.-0. was supported by Emmy Noether-Program of German Research Foundation Grant Ha4466/3-1 and German Federal Ministry of Education and Research Grant 01GQ0809. We thank Drs. C. Cozzari and B. K. Hartman for the anti-ChAT antibody and Dr. J. Staiger for helpful suggestions on histology.

Correspondence should be addressed to lleana L. Hanganu-Opatz, Developmental Neurophysiology, Center for Molecular Neurobiology, University Medical Center Hamburg-Eppendorf, Falkenried 94, 20251 Hamburg, Germany. E-mail: hangop@zmnh.uni-hamburg.de.

DOI:10.1523/JNEUROSCI.2644-11.2011

Copyright $\odot 2011$ the authors $\quad 0270-6474 / 11 / 3117955-16 \$ 15.00 / 0$
Simultaneous recordings from both areas demonstrated that hippocampal theta oscillations control the firing of prefrontal neurons, thereby delivering the temporal coordination of oscillating networks accounting for information transfer and storage (Siapas and Wilson, 1998; Sirota et al., 2008; Wierzynski et al., 2009). The activating cholinergic drive directly interacts with this oscillatory activity by modulating the hippocampal theta rhythm and shifting the neocortical activity from slow to fast oscillations (Buzsáki et al., 1983; Metherate et al., 1992; Manns et al., 2000).

Anatomically, the ascending cholinergic system is well positioned for a modulatory role. The abundant cholinergic projections to the cortex mainly originate from the basal forebrain nuclei (BFn) that lie as a continuum extending from the medial septum (MS), vertical (VDB), and horizontal (HDB) limb of the diagonal band through the nucleus basalis $(\mathrm{nB})$ to the globus pallidus (Rye et al., 1984; Semba and Fibiger, 1989). The cholinergic afferents enter at approximately birth the primary sensory cortices and slightly later the Hipp (Matthews et al., 1974; Linke and Frotscher, 1993; Mechawar et al., 2002), in which ACh exerts both trophic and modulatory function. ACh contributes to the wiring of neuronal networks and facilitates the neuronal plasticity as well as the formation of early cortical architecture (Bear and Singer, 1986; Hanganu and Luhmann, 2004; Kuczewski et al., 2005; Dupont et al., 2006; Hanganu et al., 2007).

Despite consistent data supporting the role of cholinergic modulation for the activity-dependent maturation of sensory systems, it remains unknown how the cholinergic input interacts with the development of prefrontal-hippocampal networks. We showed recently that hippocampal theta bursts drive the discontinuous activity of the neonatal PFC, whereas theta-gamma 
rhythms entrain the prejuvenile prefrontal-hippocampal networks (Brockmann et al., 2011). Here, we combined in vivo recordings from the neonatal and prejuvenile PFC and Hipp with pharmacological and immunohistochemical investigation as well as immunotoxic lesion. We provide evidence that the cholinergic projections facilitate the local coupling of prefrontal networks in gamma rhythms and modulate the early activity within prefrontal-hippocampal networks.

\section{Materials and Methods}

Surgical preparation. All experiments were performed in compliance with the German laws and the guidelines of the European Community for the use of animals in research and were approved by the local ethical committee. Pregnant Wistar rats were obtained at 14-17 d of gestation from the animal facility of the University Medical Center Hamburg-Eppendorf, housed individually in breeding cages with a $12 \mathrm{~h}$ light/dark cycle, and fed ad libitum. Extracellular recordings were performed in the PFC (2-2.5 $\mathrm{mm}$ anterior to bregma and $0.2-0.7 \mathrm{~mm}$ from the midline) and intermediate Hipp (3-4 mm posterior to bregma and 3-4 $\mathrm{mm}$ from the midline) of postnatal day 0 (P0) to P14 male rats (Ashwell and Paxinos at al., 2008) using experimental protocols as described previously (Hanganu et al., 2006; Yang et al., 2009; Brockmann et al., 2011). Briefly, under light urethane anesthesia (0.5-1 g/kg; Sigma-Aldrich), the head of the pup was mounted into the stereotaxic apparatus (Stoelting) using two metal bars set in place with dental cement on the nasal and occipital bones, respectively. The bone over the PFC and Hipp was carefully removed by drilling holes of $<0.5 \mathrm{~mm}$ in diameter. Removal of the underlying dura mater by drilling was avoided, because leakage of CSF or blood attenuates the cortical activity and single neuronal firing (I.L.H.-O., unpublished observations). The body of the animals was surrounded by cotton and kept at a constant temperature of $37^{\circ} \mathrm{C}$ by placing it on a heating blanket. After a 20-60 min recovery period, multielectrode arrays (Silicon Michigan probes; NeuroNexus Technologies) were inserted perpendicularly to the skull surface into the PFC until a depth of $3 \mathrm{~mm}$ and perpendicularly or at $20^{\circ}$ from the vertical plane into the Hipp at a depth of 2.5-3.5 mm. The electrodes were labeled with $1,1^{\prime}$-dioctadecyl$3,3,3^{\prime}, 3^{\prime}$-tetramethyl indocarbocyanine (DiI) (Invitrogen) to enable postmortem the reconstruction of electrode tracks in histological sections (see Figs. $2 A, 4 D$ ). One or two silver wires were inserted into the cerebellum and served as ground and reference electrodes. Miniature earphones placed under the pup's body were sensitive enough to detect the smallest visible movements of the limbs as well as the breathing of pups during recordings.

Recording and stimulation protocols. Simultaneous recordings of field potential (FP) and multiple-unit activity (MUA) were performed from the PFC and Hipp using one-shank 16-channel Michigan electrodes (0.5-3 $\mathrm{M} \Omega$ ). The recording sites were separated by 50 or $100 \mu \mathrm{m}$ in vertical direction. The recording sites covered in the PFC the anatomical subdivisions cingulate cortex $(\mathrm{Cg})$ and prelimbic cortex $(\mathrm{PL})$ (Van Eden and Uylings, 1985) and in the intermediate Hipp the CA1 region (see Figs. 2A, 4D). Both FP and MUA were recorded for at least $900 \mathrm{~s}$ at a sampling rate of $32 \mathrm{kHz}$ using multichannel extracellular amplifiers (Digital Lynx 4S from Neuralynx or USB-ME16-FAI System with gain of 1000 from Multichannel Systems) and the corresponding acquisition software (Cheetah or MC-RACK). Stimulation of cholinergic nuclei was performed at stereotaxic coordinates $(0-0.4 \mathrm{~mm}$ posterior to bregma, $1.5 \mathrm{~mm}$ lateral to midline, and $4-5 \mathrm{~mm}$ ventral to the pial surface) corresponding to the DiI staining of the stimulation electrode (see Fig. $2 D$ ) and to our previous results (Hanganu et al., 2007). Single or trains of electrical pulses $(0.3-0.5 \mathrm{~V}$, for repeated stimuli: 10 stimuli at 10 or 100 $\mathrm{Hz}$, every $30 \mathrm{~s}$ ) were applied via a bipolar tungsten electrode (4 M $\Omega$, tip separation of $0.4 \mathrm{~mm}$; FHC) inserted into the BFn. The occurrence and intrinsic properties of all oscillations occurring between the trains of stimuli were averaged and compared with the nonstimulated conditions.

Pharmacological procedures. Pharmacological modification of the muscarinic (mAChRs) and nicotinic (nAChRs) acetylcholine receptors was performed on pups mounted in the stereotaxic apparatus. For this purpose, a 26 gauge bent needle (WPI) attached to a microsyringe pump controller (Micro4; WPI) was used to apply $1 \mu \mathrm{l}$ of solution at a rate of $100-500 \mathrm{nl} / \mathrm{min}$ in the direct vicinity $(<0.5 \mathrm{~mm})$ of the Michigan electrode inserted into the PFC. The position of the electrode and the speed of application were chosen to avoid side effects attributable to mechanical damage of the tissue. After application, the needle was left in place for additional 1-3 min to allow the optimal diffusion of the solution. Solutions of atropine sulfate, mecamylamine hydrochloride (Sigma-Aldrich), or nicotine di-tartrate (Tocris Bioscience) were prepared on the day of the experiment in artificial CSF (ACSF) containing the following (in $\mathrm{mm}$ ): $124 \mathrm{NaCl}, 26 \mathrm{NaHCO}_{3}, 1.3 \mathrm{NaH}_{2} \mathrm{PO}_{4}, 1.9 \mathrm{MgCl}_{2}, 1.6 \mathrm{CaCl}_{2}$, and 3 $\mathrm{KCl}$, pH 7.4 (osmolarity, $333 \mathrm{mOsm}$ ). Control recordings were performed in each investigated pup before and after insertion of the injection syringe and after solvent (ACSF) injection. At the end of each injection, the needle was withdrawn and a $2-5$ min recovery period was allowed before starting the recordings.

Immunotoxin injection. Anesthetized male P0 pups were placed on a preformed mold and immobilized with tapes. A 26 gauge straight needle (WPI) attached to a microsyringe pump controller (Micro4; WPI) was used to inject at a slow rate $(0.1 \mu \mathrm{l} / \mathrm{min}) 0.5 \mu \mathrm{l}$ of $192 \mathrm{IgG}$-saporin (SAP) (Millipore; $0.2 \mu \mathrm{g} / \mu \mathrm{l}$, solved in $0.1 \mathrm{M}$ PBS, $\mathrm{pH} 7.4$ ) or $0.5 \mu \mathrm{l}$ of vehicle $(0.1$ M PBS, pH 7.4) into both lateral ventricles ( $1 \mathrm{~mm}$ posterior to bregma, 1 $\mathrm{mm}$ lateral to midline, and $2-2.5 \mathrm{~mm}$ ventral to pial surface). In some experiments, the immunotoxin or the vehicle was injected directly into the MS ( $0.1 \mathrm{~mm}$ anterior to bregma, $0.1 \mathrm{~mm}$ lateral to midline, and 3-4 $\mathrm{mm}$ ventral to the pial surface). In this case, $50 \mathrm{nl}$ of SAP $(0.5 \mu \mathrm{g} / \mu \mathrm{l})$ or PBS was injected at a slow speed $(20-50 \mathrm{nl} / \mathrm{min})$. After injection, the needle was left in place for additional 1-3 min to allow optimal diffusion of the solution. Under anesthesia, the scalp wound was closed with tissue adhesive, and the pups were tattooed on the paw or on the tail with animal tattoo ink (Raidex) using a 27 gauge needle. Pups were warmed up under a filament bulb and returned to the dam only after full recovery of body temperature and motor activity $(30-60 \mathrm{~min})$ to prevent maternal infanticide behavior. In each investigated litter, nontreated, PBStreated, and immunotoxin-treated pups were observed daily (general and feeding behavior, weight). At P7-P8 or P14-P15, the pups were anesthetized, fixed in the stereotaxic apparatus as described previously, and investigated for their prefrontal and/or hippocampal activity patterns.

Behavioral analysis. Male nontreated, PBS-treated, and SAP-treated pups originated from litters culled at birth to maximum of 12 pups. The litters included both males and females to avoid sex-based maternal behavioral biases (Alleva et al., 1989; Hahn and Lavooy, 2005). To minimize the influence of circadian rhythms, all behavioral tests were conducted during the light phase of circadian cycle. One investigator performed the entire testing to prevent interobserver variability attributable to different handling of the pups. Pups were tested according to a modified Fox battery (Fox, 1965) for their reflexes and overall growth every second day from P1 to P11 and every day from P11 to P15. Weight, body, and tail length were quantified, and the day of eyelid and ear channel opening as well as of the incisor eruption were registered. Reflexes were scored as follows: (1) righting reflex: time (in seconds) until the pup turned over with all four feet on the ground after being placed on its back; (2) forelimb grasping reflex: positive (retraction of the paw or grasping of the toothpick) or negative reaction when the forepaw was touched with a toothpick; (3) cliff aversion reflex: time (in seconds) until the pup withdraws from the edge when the snout and the forepaws are placed over the edge of a table.

Staining protocols and immunohistochemistry. For staining of cholinergic neurons in the BFn and of cholinergic projections within the PFC and Hipp as well as for their double labeling together with GABAergic neurons or vesicular transporter proteins for Glu (vGluT1, vGluT2), the rats were deeply anesthetized with $10 \%$ ketamine (aniMedica)/ $2 \%$ xylazine (WDT) in $\mathrm{NaCl}(10 \mu \mathrm{l} / \mathrm{g}$ body weight, i.p.) and perfused transcardially with $4 \%$ paraformaldehyde dissolved in $0.1 \mathrm{~m}$ phosphate buffer, $\mathrm{pH}$ 7.4. The brains were removed and postfixed in the same solution for $24 \mathrm{~h}$. Blocks of tissue containing the PFC, cholinergic nuclei, or Hipp were sectioned in either the coronal or sagittal plane at 20 or $50 \mu \mathrm{m}$. Series of two to four consecutive sections were incubated with the mouse monoclonal antibody against purified rat brain choline acetyltransferase 
(ChAT) (gift from Dr. C. Cozzari, University of Rome, Italy and Dr. B. K. Hartman, University of Minnesota, Minneapolis, MN), guinea pig polyclonal antibody against recombinant GST-tagged vGluT2 (Millipore), guinea pig polyclonal antibody against rat synthetic linear vGluT1 peptide (Millipore), rabbit polyclonal antibody against recombinant mouse calbindin (CB) (Millipore), or rabbit polyclonal antibody against muscle parvalbumin (PV) (Swant) as described previously (Umbriaco et al., 1994; Mechawar et al., 2000; Henny and Jones, 2008). Briefly, at room temperature (RT), free-floating sections were rinsed in PBS $(0.1 \mathrm{M}, \mathrm{pH}$ 7.4) and preincubated for $2 \mathrm{~h}$ in PBS containing 2\% normal horse serum (Vector Laboratories), 1\% bovine serum albumin (Roth), and $0.2 \%$ Triton X-100 (Roth) to block unspecific staining. Overnight incubation with the anti-ChAT (1:250), anti-vGluT1 (1:2000), anti-vGluT2 (1: 2000), anti-PV (1:1000), or anti-CB (1:100) antibodies was followed by rinse in PBS and incubation for $90 \mathrm{~min}$ with the secondary Alexa Fluor488 goat anti-mouse IgG, Alexa Fluor-546 goat anti-mouse IgG, or Alexa Fluor-546 goat anti-guinea pig antibodies (Invitrogen). Sections were then rinsed several times in PBS at RT and counterstained with Hoechst 3342 dye (1:1000; Invitrogen). The sections were air dried, coverslipped with Fluoromount G (Biozol), and examined using the 380, 488, or 546 $\mathrm{nm}$ excitation filters of a Carl Zeiss Imager M1 microscope. The photographs were adjusted for brightness and contrast using Adobe Photoshop CS4 (Adobe Systems). For quantification, 20- $\mu \mathrm{m}$-thick coronal slices including the PFC from three P7-P8 and three P14 rats were used, and 30 CB-positive cells per slice were tested for their proximity to ChATpositive fibers.

Fluorescent Nissl staining was performed as described previously (Quinn et al., 1995) using the NeuroTrace 500/525 green fluorescent Nissl stain (Invitrogen). Briefly, rehydrated slices were incubated for 20 min with 1:100 diluted NeuroTrace. Sections were washed, coverslipped with Fluoromount, and examined using the green filter (AF 488) of the fluorescence microscope (Imager M1; Carl Zeiss).

Receptor autoradiography and binding studies. Deeply anesthetized rat pups were killed by decapitation. The brains were quickly removed, shock frozen in liquid nitrogen, and stored at $-80^{\circ} \mathrm{C}$. Coronal sections of $20 \mu \mathrm{m}$ thickness were cut on a freezing microtome, mounted onto Superfrost slides, air dried, and kept at $-20^{\circ} \mathrm{C}$ until use. $\left[{ }^{125} \mathrm{I}\right]$ Epibatidine binding was performed as described previously (Perry et al., 1999). Briefly, the sections were preincubated with Tris-HCl-buffered solution (50 nM Tris- $\mathrm{HCl}, \mathrm{pH} 7.4,120 \mathrm{~mm} \mathrm{NaCl}$ ) for $10 \mathrm{~min}$ at RT and subsequently incubated in the same buffer in the presence of $0.4 \mathrm{nM}\left[{ }^{125} \mathrm{I}\right]$ epibatidine (PerkinElmer Life and Analytical Sciences) for $1 \mathrm{~h}$ at RT. Nonspecific binding was determined in adjacent sections in the presence of $200 \mu \mathrm{M}$ nicotine di-tartrate included in preincubation and incubation solutions. Sections were washed three times for $5 \mathrm{~min}$ in ice-cold distilled water and air dried at RT. Slides were exposed to Hyperfilm MP (GE Healthcare). For $\alpha$-bungarotoxin binding, the slides were preincubated with the Tris-HCl-buffered solution for $10 \mathrm{~min}$ at RT and subsequently incubated with the same buffer containing Alexa Fluor-555 $\alpha$-bungarotoxin (1:500; Invitrogen) for $2 \mathrm{~h}$ at RT. Nonspecific binding was determined in adjacent sections in the presence of $200 \mu \mathrm{M}$ nicotine di-tartrate included in preincubation and incubation solutions. Sections were rinsed three times for $5 \mathrm{~min}$ in ice-cold distilled water, with Hoechst 3342 dye (1:1000) added to the first wash for counterstaining. The slides were air dried, coverslipped using Fluoromount, and examined using the $546 \mathrm{~nm}$ excitation filter of the fluorescence microscope (Imager M1; Carl Zeiss).

Data analysis. Data were imported and analyzed offline using customwritten tools in Matlab software version 7.7 (MathWorks). To detect the oscillatory events, the raw data were filtered between 4-30, 30-100, or $100-300 \mathrm{~Hz}$ using a Butterworth three-order filter. Discontinuous or continuous slow oscillations in theta-alpha frequency band were detected as FP deflections exceeding five times the baseline SD. Only discontinuous slow events lasting $>100 \mathrm{~ms}$, containing at least three cycles, and being not correlated with movement (twitches) were considered for analysis. For continuous activity, movement-associated epochs were removed. Fast events (gamma episodes in the PFC, gamma oscillations and ripples in the Hipp) superimposed on slow events were detected either automatically or by eye and confirmed by time-frequency plots. Spindle bursts (SB) and nested gamma spindle bursts (NG) were classified according to previously described criteria (Khazipov et al., 2004; Brockmann et al., 2011). The presence of short gamma episodes automatically detected after $30-100 \mathrm{~Hz}$ filtering and confirmed by eye on at least onethird of the cycles in intermittent slow oscillations led to classification of these events as NG. Intermittent oscillatory events, SB and NG in the PFC and theta bursts in the hippocampal CA1 area, were analyzed in their occurrence (defined as the number of bursts per minute), duration, maximum amplitude (defined as the voltage difference between the maximal positive and negative peaks), dominant frequency, as well as absolute and relative power in theta (cortical, $4-8 \mathrm{~Hz}$; hippocampal, $4-10 \mathrm{~Hz}$ ), alpha (cortical, $8-13 \mathrm{~Hz}$ ), and beta (cortical, 13-30 Hz) frequency bands. For fast discontinuous gamma oscillations $(30-100 \mathrm{~Hz})$, the duration and dominant frequency were investigated. Oscillatory activity was defined and analyzed as continuous if the average interburst interval was below $0.5 \mathrm{~s}$. For continuous oscillatory activity, same parameters as for slow intermittent events, except occurrence and duration, were analyzed for 5-15 consecutive 60-s-long time windows. Time-frequency plots were calculated by transforming the FP events using Morlet continuous wavelet. Minimal and maximal intensities in power were normalized to values between 0 and 1 and were displayed in dark blue and red, respectively. For visualization of data, "whitened" spectrograms were calculated as described previously (Sirota et al., 2008).

Prefrontal and hippocampal oscillations were termed simultaneous if their onset difference was $<13 \mathrm{~ms}$. Maximal cross-correlation and coherence coefficients of FP were calculated for all recording sites by using the hippocampal signal as reference. As spectral measure of correlation between two signals across frequencies, the coherence was calculated from the cross-spectral density between the two signals and normalized by the power spectral density of each (Jerbi et al., 2007). The computation was performed according to the following formula:

$$
C(f)=\frac{\left|\sum_{i=1}^{N} X_{i}(f) Y_{i}^{*}(f)\right|^{2}}{\sum_{i=1}^{N}\left|X_{i}(f)\right|^{2} \sum_{i=1}^{N}\left|Y_{i}(f)\right|^{2}}
$$

where $X_{i}(f)$ and $Y_{i}(f)$ are the Fourier transforms of the signals $x$ and $y$ for the $i$ data segment at frequency $f$, and ${ }^{*}$ indicates the complex conjugate. The computations were performed using the magnitude-squared coherence function (Matlab) based on Welch's averaged periodogram method (non-overlapping $0.5 \mathrm{~s}$ time window, frequency resolution of $1 \mathrm{~Hz}$ ).

Statistics. Data in the text are presented as mean \pm SEM. Statistical analyses were performed with SPSS 15.0/Systat software (SPSS). All values were tested for normal distribution by the Kolmogorov-Smirnov test. For normally distributed values, paired or unpaired $t$ test was used to detect significance levels $\left({ }^{*} p<0.05,{ }^{* *} p<0.01\right.$, or $\left.{ }^{\star * *} p<0.001\right)$. Values that were not normally distributed were tested by nonparametric tests (Mann-Whitney-Wilcoxon test).

\section{Results}

\section{Ingrowing cholinergic projections from the basal forebrain target GABAergic neurons in the neonatal and prejuvenile PFC}

To get a first insight into the role of cholinergic innervation for the maturation of prefrontal networks, we characterized the developmental profile of ingrowing projections from the cholinergic BFn to the PFC during the first 2 postnatal weeks. To avoid the influence of different maturation dynamics in males and females of the cholinergic system (Muñoz-Cueto et al., 1991; Arters et al., 1998), only male pups were used for all investigations. Immunohistochemical staining for ChAT performed at P0, P6, and $\mathrm{P} 13$ ( $n=18$ pups) on coronal and sagittal sections including the PFC revealed the progressive densification of cholinergic axons with age. Although ChAT-positive projections cannot be de- 

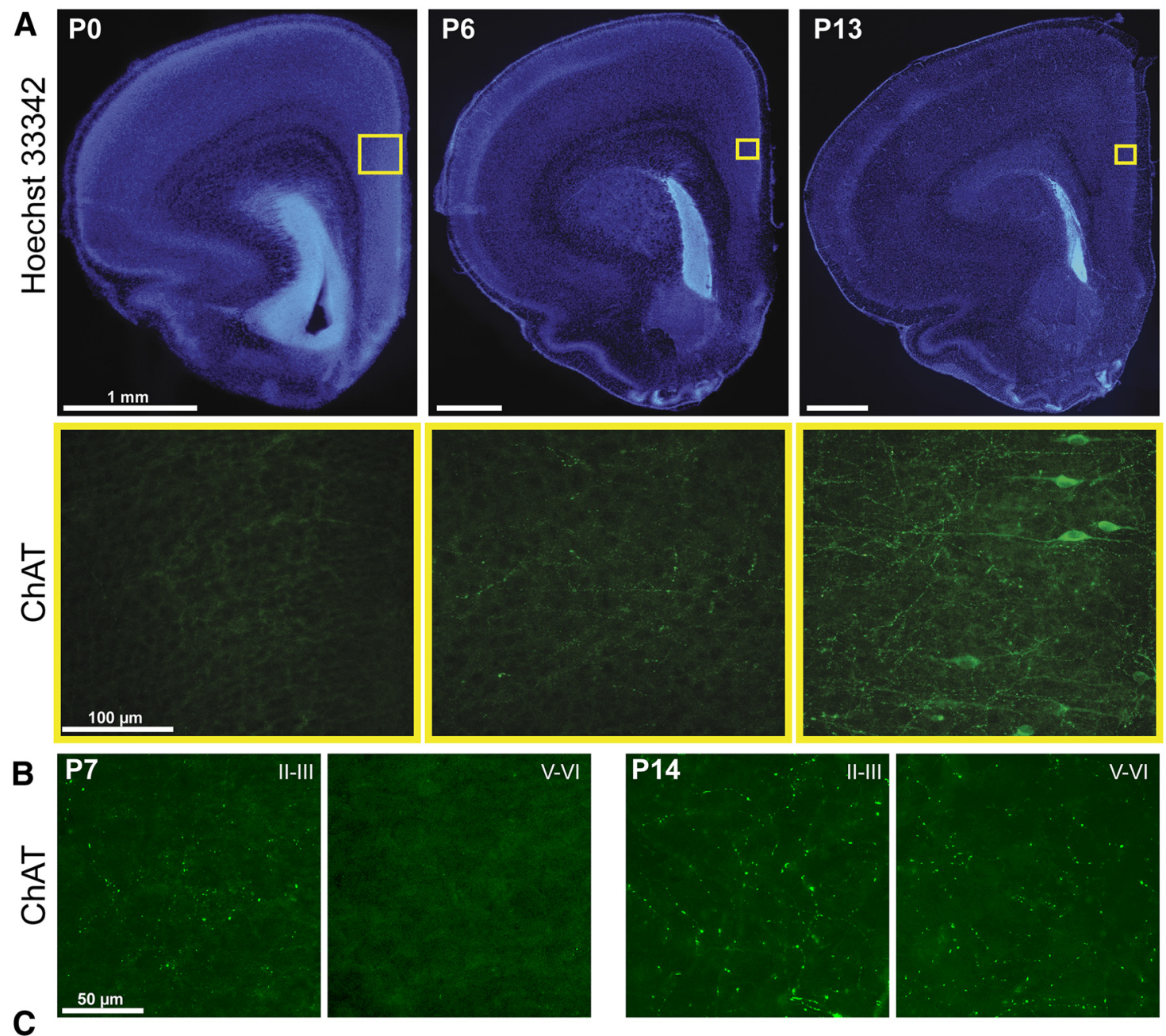

(i)
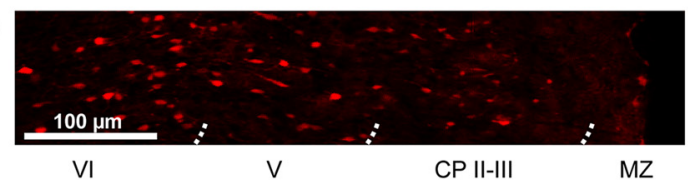

CP II-III

$M Z$
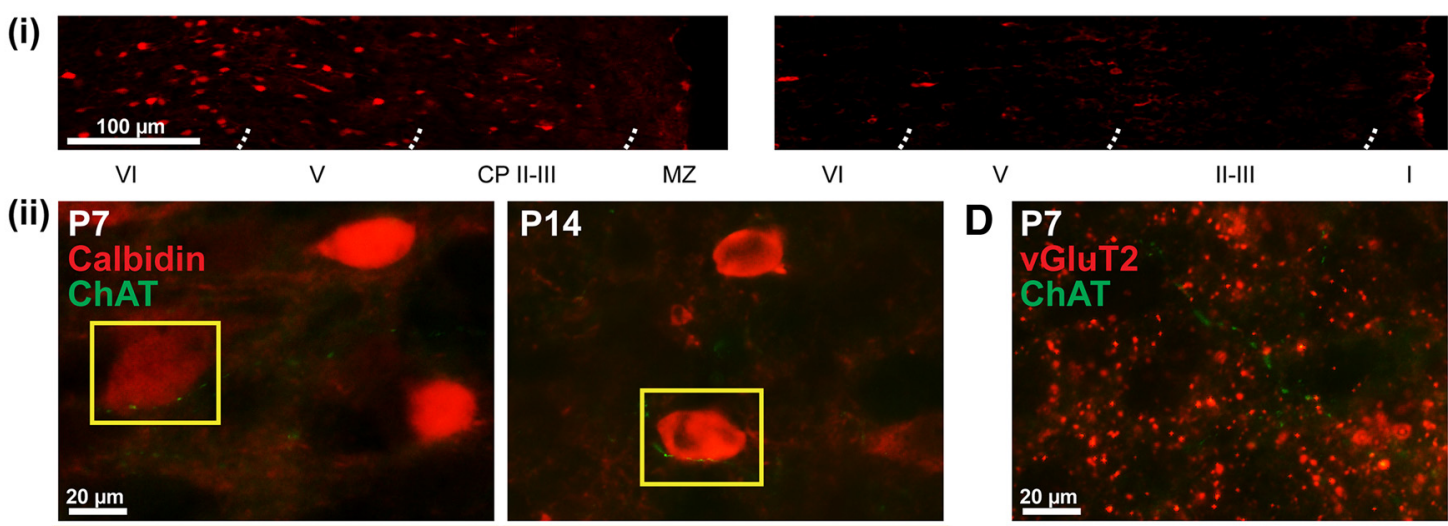

D P7
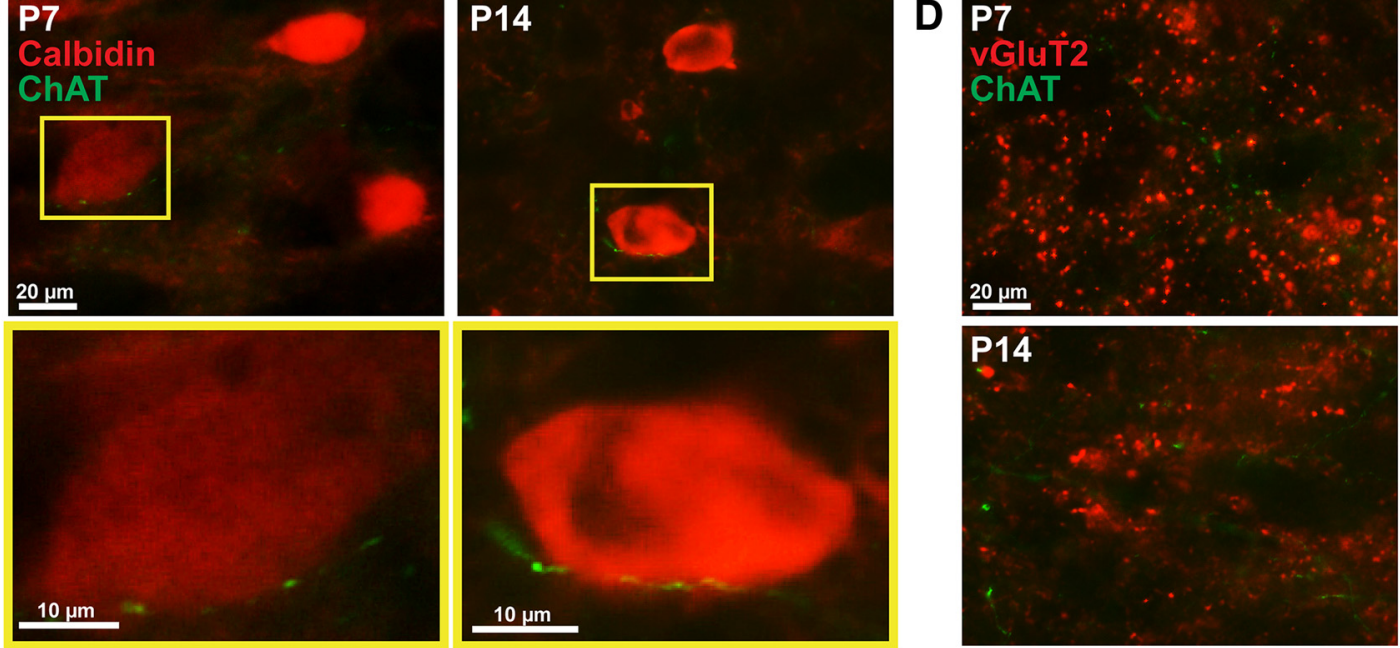

Figure 1. Developmental profile of ingrowing cholinergic projections into the neonatal and prejuvenile PFC. $A$, Nuclear Hoechst 33342 (blue, top row) combined with immunofluorescent ChAT staining (green, bottom row) of 100- $\mu \mathrm{m}$-thick coronal sections from P0, P6, and P13 rats, respectively. Note the absence of ChAT-positive projections in the PFC at birth, their densification with age, and the presence of prefrontal cholinergic cells toward the end of the second postnatal week. Yellow squares in the top row correspond to the prefrontal area at the border of the C $g$ and PL that is shown below for ChAT staining. $\boldsymbol{B}$, Density of the ChAT fibers in the CP and layers II/III as well as in layers V/NI at the end of the first and second postnatal week. C, Relationship between cholinergic projections and CB-positive GABAergic neurons in the neonatal and prejuvenile PFC. Ci, Distribution of CB-positive GABAergic neurons over the PFC depth at P7 and P14. MZ, Marginal zone. Cii, Double staining of ChAT projections (Figure legend continues.) 
tected in the PFC at approximately birth, scattered low-density axons appeared in the cortical plate $(\mathrm{CP})$ and layers $\mathrm{V}$ and $\mathrm{VI}$ toward the end of the first postnatal week (Fig. 1A). Similar dynamics of cholinergic innervation characterized the two anatomical subdivisions of the PFC, the $\mathrm{Cg}$, and the PL. With ongoing maturation, the density of cholinergic projections increased in all layers but particularly in the upper layers II/III (Fig. $1 B$ ). Additionally, few ChAT-positive cells, which have been described previously (Eckenstein et al., 1988; Lysakowski et al., 1989) but the function of which is still unknown, could be detected in the prejuvenile PFC (Fig. $1 A$ ). Their projections represent $<10 \%$ of the total amount of ChAT fibers (Vaucher and Hamel, 1995), and, therefore, these intracortical cholinergic neurons most likely do not account for the increased density of cholinergic projections in prejuvenile rats.

To identify the targets of cholinergic projections into the PFC, double stainings for ChAT and GABAergic markers CB and PV, as well as for ChAT, vGluT1, and vGluT2, were performed (Fig. $1 C, D)$. The low expression of PV and vGluT1 in the neonatal PFC precluded additional investigation. In contrast, a large number of CB-positive cells were present in both the neonatal and prejuvenile PFC, although their density decreased toward the end of the second postnatal week (Fig. 1Ci). Investigation of coronal sections stained for both ChAT and CB revealed that, throughout the entire development, cholinergic projections run very closely and seem to contact a fraction $(31.3 \pm 3.3 \%$ in P7-P8 rats, $23.1 \pm$ $1.3 \%$ in P14 rats) of prefrontal CB-positive cells (Fig. 1Cii). The opposite was observed when double staining for ChAT and vGluT2 was performed (Fig. $1 D$ ). The vGluT2 varicosities distributed over the layers of neonatal and prejuvenile PFC were not spatially correlated with ChAT-stained fibers. Although the cellular interactions between cholinergic projections and different types of prefrontal neurons remain to be investigated in detail, these preliminary data suggest that the ingrowing projections initially target GABAergic neurons in the developing PFC.

\section{Activation of cholinergic projections differentially modulates} the dominant activity patterns of the neonatal $\mathrm{Cg}$ and $\mathrm{PL}$

To decide whether the early cholinergic innervation acting mainly on CB-positive GABAergic neurons influences the patterns of prefrontal oscillatory activity, we electrically stimulated the cholinergic BFn and simultaneously recorded the FP and MUA in the PFC of P7-P8 rat pups $(n=13)$ (Fig. $2 A)$. We recently characterized in detail the spontaneous network activity of the rat PFC during the first 2 postnatal weeks (Brockmann et al., 2011). At the arrival of the first cholinergic axons, the PFC shows discontinuous patterns of oscillatory activity (Fig. 2B) that, according to their properties, have been classified previously as SB, intermittent spindle-shape field oscillations with main frequency within theta band (Fig. 2Ci), and as NG, theta-alphaband network oscillations superimposed with short phase-locked oscillations in gamma frequency band (Fig. 2Cii). These patterns of activity show distinct spatial and temporal organization as well as current generators over the Cg and PL. As shown previously,

\section{$\leftarrow$}

(Figure legend continued.) (green) and (B-positive neurons (red) at P7 and P14. CB-positive neurons contacted by cholinergic projections are depicted in the bottom row at higher magnification. $\boldsymbol{D}$, Relationship between cholinergic projections and glutamatergic neurons in the neonatal and prejuvenile PFC. Double staining of ChAT projections (green) and vGluT2 (red) at P7 and P14.
SB predominate in the $\mathrm{Cg}$, whereas NGs have the highest occurrence in the prelimbic networks, in which they are also generated.

Stimulation of the BFn was performed as described previously using stereotaxic coordinates (Hanganu et al., 2007; Ashwell and Paxinos, 2008) that were confirmed by DiI labeling of the electrodes (Fig. 2 D). The deep insertion of the stimulation electrode required by the ventral localization of the BFn may have damaging effects on several brain structures with more dorsal position and, therefore, indirectly on the prefrontal activity. To decide on the consequences of such unspecific lesion, we investigated the oscillatory activity of the PFC before and after insertion of the stimulation electrode $(n=5$ pups). The occurrence of SB in the $\mathrm{Cg}$ and PL was comparable before $(3.38 \pm 0.58$ and $3.61 \pm 0.35$ bursts/min) and after $(2.88 \pm 0.3$ and $2.95 \pm 0.36$ bursts $/ \mathrm{min})$ insertion. The same lack of effects was observed for the occurrence of NGs in the Cg (before, $0.2 \pm 0.11$ bursts $/ \mathrm{min}$; after, $0.06 \pm 0.04$ bursts $/ \mathrm{min}$ ) and PL (before, $0.5 \pm 0.1$ bursts $/ \mathrm{min}$; after, $0.34 \pm 0.1$ bursts $/ \mathrm{min}$ ) and for the intrinsic properties (amplitude, duration, dominant frequency) of SB and NG. Only the amplitude of cingulate NG decreased significantly $(p=0.02)$ from $189.46 \pm 48.18$ to $108.88 \pm 22.05 \mu \mathrm{V}$ after electrode insertion.

Single electrical stimulation of the $\mathrm{nB}$ performed every $30 \mathrm{~s}$ elicited oscillatory discharge in the $\mathrm{Cg}$ and PL of three of five investigated pups. These oscillations started shortly after the stimulus onset $(\mathrm{Cg}, 0.53 \pm 0.05 \mathrm{~s} ; \mathrm{PL}, 0.57 \pm 0.05 \mathrm{~s})$ and had high amplitude (Fig. 2E). Because of the immaturity of cholinergic innervation, the failure rate of stimulation was also high, with only $\sim 27 \%$ of stimulations evoking oscillatory discharge. To increase the efficiency of stimulation and mimic the ACh release in vivo that correlates with the bursting firing at $10 \mathrm{~Hz}$ of $\mathrm{nB}$ cholinergic neurons (Khateb et al., 1992; Alonso et al., 1996), we used the previously described tetanic stimulation protocol (Metherate and Ashe, 1991; Rasmusson et al., 1994; Hanganu et al., 2007). Repetitive stimulation of the BFn at $10 \mathrm{~Hz}$ for $1 \mathrm{~s}$ performed every $30 \mathrm{~s}$ for 20-30 times was defined as stimulation epoch. Because very few, if any, cholinergic projections are present in the PFC before $\mathrm{P7}$, the stimulation protocol was not applied to rats younger than P7. Tetanic stimulation of the BFn had different effects on the oscillatory activity of the $\mathrm{Cg}$ and PL (Fig. $2 \mathrm{~F}$ ). Although BFn stimulation did not modify the properties of cingulate SB and NG, it increased significantly $(p=0.043)$ the occurrence of prelimbic NG from $0.34 \pm 0.1$ bursts $/$ min $(n=5$ pups) after insertion of the stimulation electrode to $0.48 \pm 0.13$ bursts/min during the stimulation epoch. Moreover, the duration of prelimbic SB decreased from $2.93 \pm 0.22$ to $2.15 \pm 0.17 \mathrm{~s}$ $(p=0.038)$. These data suggest that, despite the equal presence of cholinergic axons able to evoke oscillatory activity in both $\mathrm{Cg}$ and $\mathrm{PL}$, their repetitive electrical activation at frequencies $(\sim 10 \mathrm{~Hz})$ similar to neuronal bursting in the $\mathrm{nB}$ in vivo predominantly modulates the prelimbic NG.

\section{Immunotoxic lesion of all cholinergic nuclei modifies the oscillatory activity of the developing PL}

The cholinergic forebrain innervation controls not only the adult cortical structure and function $(\mathrm{Gu}, 2002)$ but also the activity-dependent maturation of the primary sensory cortices (Höhmann, 2003; Hanganu et al., 2007). To decide on its role for the developing PFC, we analyzed the effects of long-lasting impairment of cholinergic neurons on the oscillatory activity of the $\mathrm{Cg}$ and PL. For this purpose, we used a selective method to permanently lesion the cholinergic neurons of all BFn. In contrast to electrolytic or excitatory acid-induced lesions (Höhmann and 
A
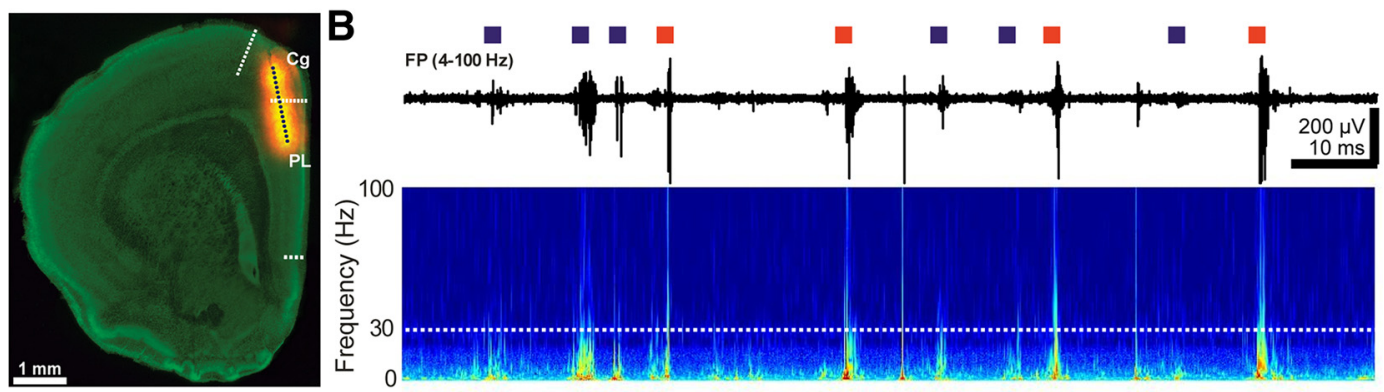

C

(i)

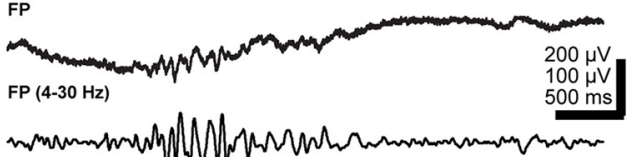

(ii)
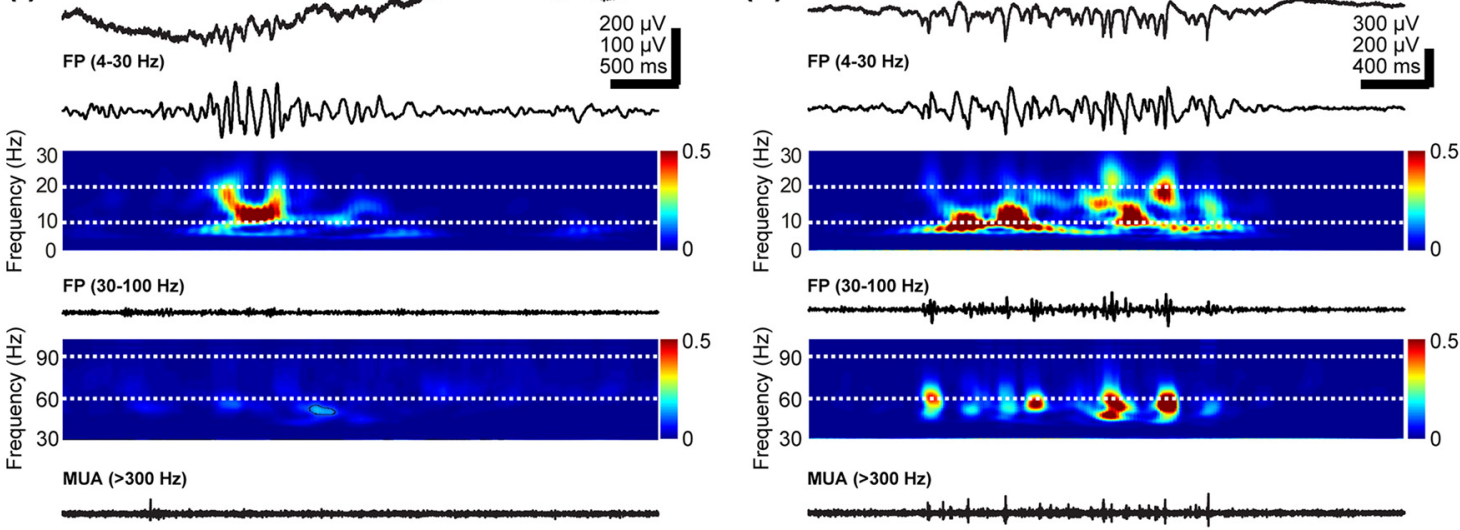

D

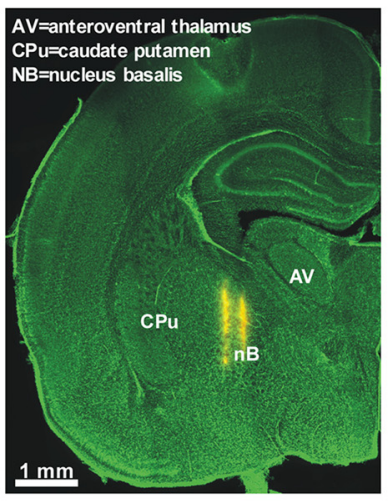

E

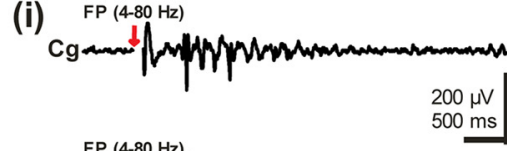

PL FP (4-80 Hz)
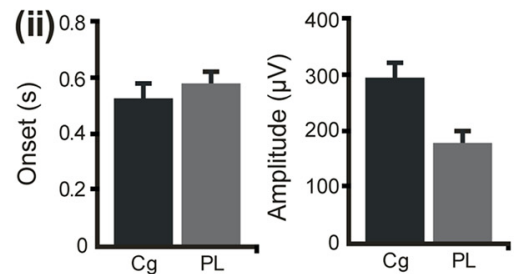

F

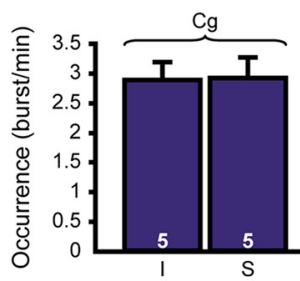

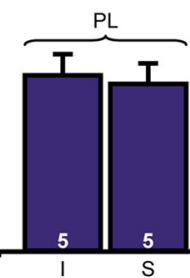
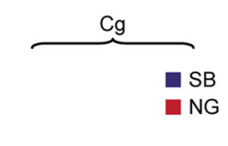

Figure 2. Effects of cholinergic activation on the patterns of network activity in the neonatal PFC. $A$, Digital photomontage reconstructing the location of the Dil-covered recording electrode (orange) in the PFC of a Nissl-stained 50- $\mu$ m-thick coronal section (green) from a P7 rat. Blue dots mark the 16 recording sites covering the cingulate and the prelimbic areas of the PFC. $\boldsymbol{B}$, Extracellular FP recording of the intermittent oscillatory activity in the PFC of a P7 rat. Blue squares mark the SB, whereas red squares correspond to NG. The trace is accompanied by the "whitened" spectrogram of FP at identical timescale. The white dotted line marks the lower border of gamma frequency band ( $30 \mathrm{~Hz}$ ). Note the wider frequency distribution for NG than for SB. Ci, Characteristic SB displayed before (top) and after bandpass (4-30 and 30-100 Hz) filtering (middle) and the corresponding MUA after $300 \mathrm{~Hz}$ high-pass filtering. Note the low spike discharge during SB. Color-coded frequency plots shows the wavelet spectrum of the FP with a mean frequency in alpha band and without detectable gamma activity. Cii, Characteristic NG displayed before (top) and after bandpass (4-30 and 30-100 Hz) filtering (middle) and the corresponding MUA after $300 \mathrm{~Hz} \mathrm{high-pass} \mathrm{filtering.} \mathrm{Note} \mathrm{the} \mathrm{presence} \mathrm{of} \mathrm{prominent} \mathrm{gamma} \mathrm{episodes} \mathrm{that} \mathrm{are} \mathrm{accompanied} \mathrm{by} \mathrm{spike}$ discharge. The top color-coded wavelet spectrum reveals the main frequency of NG within the theta-alpha band, whereas the NG episodes appear as periodic high-power spots on the bottom wavelet. D, Digital photomontage reconstructing the location of Dil-covered bipolar stimulation electrode (yellow) in a 100 - $\mu \mathrm{m}$-thick Nissl-stained coronal section, including the $n B$. Ei, Examples of SB elicited in the C $g$ and PL by single electrical stimulation of the $n B$. Red arrows mark the electrical stimulus. Stimulus artifacts were removed. Eii, Properties of SB evoked in the C $g$ and PL after single stimulation of the $\mathrm{nB}$. Bar diagrams depict the similar onset (left) and the significantly $(p<0.05)$ different amplitude (right) of evoked oscillations in the two prefrontal areas. $\boldsymbol{F}$, Bar diagram displaying the occurrence of SB and NG in the $\mathrm{g} g$ and PL of five P7-P8 pups after electrode insertion (I) and tetanic stimulation of the $\mathrm{nB}(\mathrm{S})$.

Coyle, 1988), with an inherent lack of sensitivity, immunolesion using SAP led to exclusive destruction of cholinergic neurons in the BFn by recognizing the expressed p75 receptor (Wiley et al., 1991; Book et al., 1992; Robertson et al., 1998; Pappas and Sherren, 2003).

When intracerebroventricularly applied, SAP impaired the cholinergic neurons in all BFn (Fig. $3 A$ ). The consequences of progressive SAP-induced cholinergic depletion were investigated in 32 pups from seven litters at two developmental stages, the end of the first (P7-P8) and of the second (P14-P15) postnatal week. As shown by our previous results, the prefrontal SB and NG reaching maximal amplitude and occurrence at approximately P7-P8 are replaced during the second postnatal week by continuous oscillatory rhythms with dominant frequency in theta band 
A

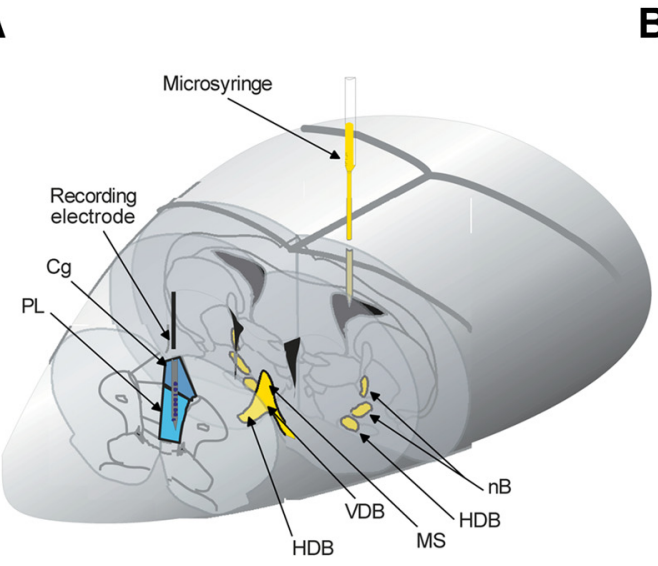

D

(i)

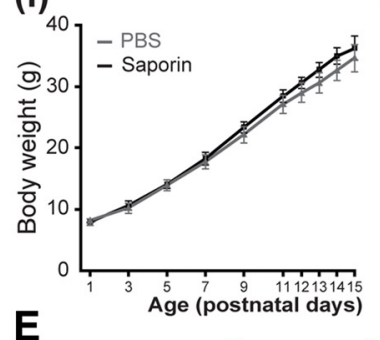

(ii)

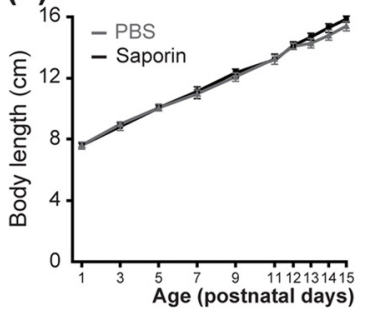

(i)

PBS

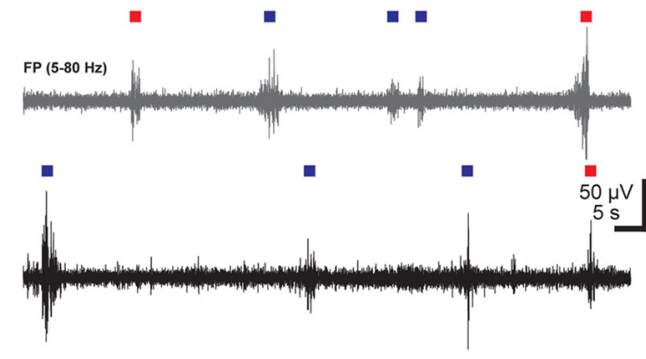

F

(i)

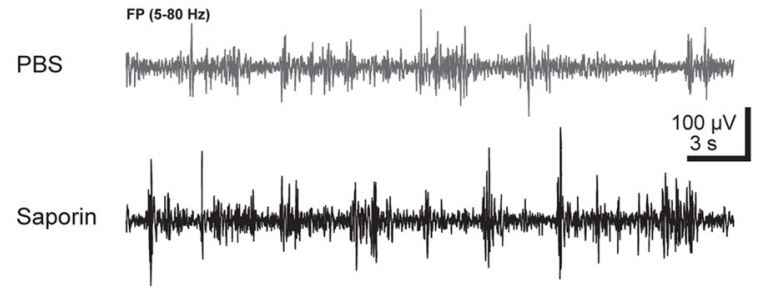

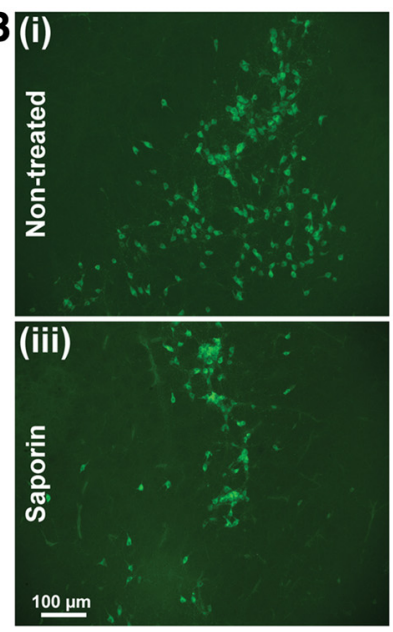

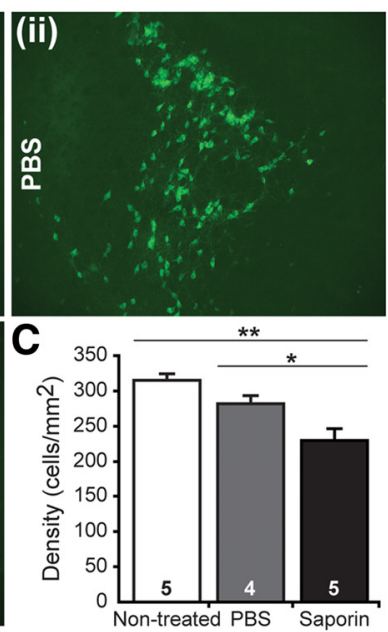

(iii)

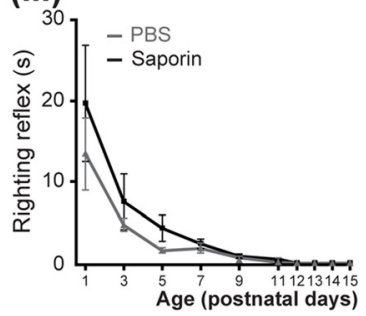

(iv)
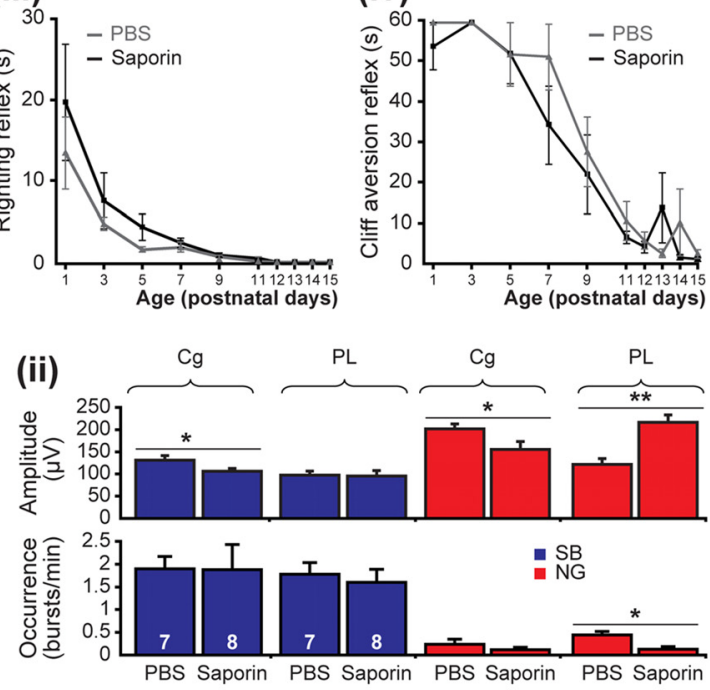

(ii)

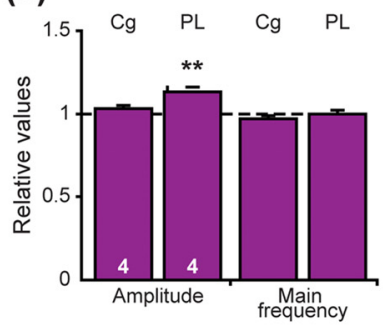

Figure 3. Consequences of global cholinergic depletion by SAP on the prefrontal network activity and the developmental milestones of rat pups. $A$, Scheme of experimental setup for recording the PFC (blue) and impairing all cholinergic nuclei by intracerebroventricular injection of SAP (yellow) at P0. B, Immunohistochemistry of ChAT-positive neurons of nontreated (i), PBS-treated (ii), and SAP-treated (iii) pups. The pups were intracerebroventricularly injected at P0 and investigated at P7. Note the decreased number of cholinergic neurons in SAP-treated pups when compared with nontreated or PBS-treated rats. C, Bar diagram displaying the density of (hAT-positive neurons under nontreated conditions (white), after PBS treatment (gray), and after SAP lesion (black). The number of investigated pups is marked on the corresponding bar. $\boldsymbol{D}$, Developmental profile of somatic growth [body weight (i), body length (ii)] and reflexes [righting reflex (iii), cliff aversion reflex (iv)] in PBS-treated (gray) and SAP-treated (black) pups. E, Consequence of SAP treatment on the prefrontal patterns of neonatal activity. Ei, Extracellular FP recordings from the PL of a P7 PBS-treated pup (gray) and a P7 SAP-treated pup (black). Blue and red squares differentiate between SB and NG. Eii, Bar diagram displaying the mean amplitude (top) and occurrence (bottom) of SB (blue) and NG (red) in the (g and PL of seven PBS-treated and eight SAP-treated pups. Fi, Extracellular FP recordings from the PL of a P14 PBS-treated pup (gray) and a P14 SAP-treated pup (black). Fii, Bar diagram displaying the amplitude and mean frequency of continuous activity in the ( $g$ and PL of four SAP-lesioned pups normalized to the properties of PBS-treated pups (dotted line).

(4-8 Hz), over which low-amplitude short gamma episodes are superimposed (Brockmann et al., 2011). All investigated rats were divided into three groups: (1) nontreated pups $(n=9),(2)$ pups receiving a bilateral injection of a total of $1 \mu \mathrm{l}$ of PBS (solvent for SAP) at P0 $(n=11)$ that served as control, and (3) pups receiving a bilateral injection of a total of $1 \mu \mathrm{l}$ of SAP $(0.2 \mu \mathrm{g} / \mu \mathrm{l})$ in PBS at P0 $(n=12)$. As comprehensively characterized in previous studies (Robertson et al., 1998), SAP treatment led to massive and progressive reduction in the number of BFn cholinergic neurons in the SAP-treated pups when compared with the nontreated pups, whereas no significant changes were observed after PBS injection (controls) (Fig. 3B). Quantification of the neuronal 
Table 1. Properties of neonatal SB and NG as well as of prejuvenile continuous theta- gamma oscillations recorded in the $\mathrm{Cg}$ and PL of the control (intracerebroventricular injection of PBS) and cholinergically impaired (intracerebroventricular injection of SAP) rats

\begin{tabular}{|c|c|c|c|c|c|c|c|c|c|c|c|c|}
\hline \multirow[b]{4}{*}{ Properties } & \multicolumn{8}{|c|}{ Discontinuous activity (P7-P8) } & & & & \\
\hline & \multicolumn{4}{|l|}{$\mathrm{Cg}$} & \multicolumn{4}{|l|}{ PL } & \multicolumn{4}{|c|}{ Continuous activity (P14-P15) } \\
\hline & \multicolumn{2}{|l|}{ SB } & \multicolumn{2}{|l|}{ NG } & \multicolumn{2}{|l|}{ SB } & \multicolumn{2}{|l|}{ NG } & \multicolumn{2}{|l|}{$\mathrm{Cg}$} & \multicolumn{2}{|l|}{ PL } \\
\hline & PBS & Saporin & PBS & Saporin & PBS & Saporin & PBS & Saporin & PBS & Saporin & PBS & Saporin \\
\hline Occurrence (bursts/min) & $1.9 \pm 0.27$ & $1.88 \pm 0.55$ & $0.24 \pm 0.11$ & $0.12 \pm 0.05$ & $1.78 \pm 0.25$ & $1.6 \pm 0.29$ & $0.44 \pm 0.08$ & $\begin{array}{l}0.13 \pm 0.06 \\
(p=0.011)^{*}\end{array}$ & & & & \\
\hline Duration (s) & $2.74 \pm 0.15$ & $\begin{array}{l}2.28 \pm 0.12 \\
(p=0.016)^{*}\end{array}$ & $2.99 \pm 0.26$ & $\begin{array}{l}1.68 \pm 0.23 \\
(p=0.002)^{* *}\end{array}$ & $2.66 \pm 0.16$ & $\begin{aligned} 2.13 & \pm 0.17 \\
(p & =0.03)^{*}\end{aligned}$ & $2.96 \pm 0.21$ & $\begin{array}{l}1.64 \pm 0.18 \\
(p=0.0002)^{* * *}\end{array}$ & & & & \\
\hline Amplitude $(\mu \mathrm{V})$ & $132.02 \pm 9.93$ & $\begin{array}{l}106.66 \pm 6.99 \\
\quad(p=0.037)^{*}\end{array}$ & $202.74 \pm 10.89$ & $\begin{array}{c}156.03 \pm 18.74 \\
(p=0.03)^{*}\end{array}$ & $97.46 \pm 10.27$ & $96.22 \pm 12.35$ & $122.28 \pm 13.77$ & $\begin{aligned} 216.9 & \pm 17.54 \\
(p & =0.008)^{* *}\end{aligned}$ & $234.22 \pm 6.85$ & $240.7 \pm 4.94$ & $269.87 \pm 6.31$ & $\begin{array}{l}306.19 \pm 8.36 \\
\quad(p=0.001)^{* *}\end{array}$ \\
\hline Main frequency (Hz) & $6.85 \pm 0.29$ & $6.86 \pm 0.27$ & $7.69 \pm 0.66$ & $7.31 \pm 0.66$ & $6.95 \pm 0.34$ & $6.86 \pm 0.34$ & $7.43 \pm 0.49$ & $8.06 \pm 0.89$ & $5.92 \pm 0.12$ & $5.71 \pm 0.14$ & $5.75 \pm 0.13$ & $5.79 \pm 0.1$ \\
\hline Relative power theta (\%) & $36.99 \pm 1.7$ & $39.7 \pm 1.42$ & $38.6 \pm 2.31$ & $\begin{array}{l}30.34 \pm 2.75 \\
(p=0.037)^{*}\end{array}$ & $39.73 \pm 2.44$ & $40.36 \pm 2.03$ & $40.48 \pm 2.31$ & $\begin{array}{c}30.83 \pm 3.03 \\
(p=0.02)^{*}\end{array}$ & $39.87 \pm 0.62$ & $40.52 \pm 0.65$ & $36 \pm 0.9$ & $38.1 \pm 0.89$ \\
\hline Relative power alpha (\%) & $25.62 \pm 1.24$ & $24.18 \pm 0.96$ & $23.02 \pm 2$ & $26.05 \pm 2.07$ & $24.24 \pm 1.58$ & $22.19 \pm 1.25$ & $23.82 \pm 1.45$ & $22.75 \pm 1.62$ & $23.09 \pm 0.34$ & $23.38 \pm 0.29$ & $21.3 \pm 0.4$ & $\begin{array}{l}22.59 \pm 0.38 \\
(p=0.023)^{*}\end{array}$ \\
\hline Relative power beta (\%) & $24.45 \pm 1.46$ & $22.33 \pm 1.3$ & $29.97 \pm 3.6$ & $33.29 \pm 3.39$ & $22.99 \pm 2.04$ & $22.64 \pm 1.74$ & $25.59 \pm 2.51$ & $\begin{array}{l}35.66 \pm 3.49 \\
(p=0.028)^{*}\end{array}$ & $21.85 \pm 0.83$ & $21.52 \pm 0.88$ & $25.37 \pm 1.22$ & $\begin{array}{l}23.8 \pm 1.29 \\
(p=0.00001)^{* * *}\end{array}$ \\
\hline Relative power gamma (\%) & & & & & & & & & $8.51 \pm 0.31$ & $\begin{aligned} 6.84 & \pm 0.18 \\
(p & =0.00001)^{* * *}\end{aligned}$ & $10.8 \pm 0.58$ & $7.81 \pm 0.29$ \\
\hline $\begin{array}{l}\text { Main frequency in gamma } \\
\text { band }(\mathrm{Hz})\end{array}$ & & & & & & & & & $35.54 \pm 0.49$ & $35.85 \pm 0.41$ & $37.11 \pm 0.48$ & $36.85 \pm 0.66$ \\
\hline
\end{tabular}

Values are presented as mean \pm SEM, and significance values $\left({ }^{*} p<0.05,{ }^{* *} p<0.01,{ }^{* * *} p<0.001\right.$, Mann-Whitney-Wilcoxon test) are indicated in parentheses.

loss in all cholinergic nuclei at P7 showed that SAP significantly $(p=0.038)$ reduced the density of cholinergic neurons from $314.75 \pm 9.84$ cells $/ \mathrm{mm}^{2}$ in nontreated pups $(n=5)$ to $229.51 \pm$ 16.39 cells $/ \mathrm{mm}^{2}$ in SAP-treated pups $(n=5)$, whereas PBS injection had no significant effect on the number of cholinergic neurons (281.97 \pm 11.48 cells $/ \mathrm{mm}^{2}, n=4$ pups) (Fig. $3 C$ ).

Because SAP treatment may generally impair the development of pups by affecting their behavior and feeding abilities (Leanza et al., 1996; Pappas et al., 1996), we analyzed the developmental milestones and the reflexes of control and SAP-treated pups. Both groups showed similar progressive increase in body weight and length as well as in tail length during the first 2 postnatal weeks, indicating that SAP treatment did not affect the feeding abilities of pups (Fig. 3Di,Dii). Moreover, the thermoregulation maturated similarly in both groups, as shown by the comparable age-dependent decrease of the difference between the body temperature at the beginning and at the end of the investigation (data not shown). SAP-treated pups had normal reflexes, with neither the righting nor the cliff aversion (Fig. 3Diii,Div) or forelimb grasping reflex showing abnormal developmental dynamics. The similar maturation of control and SAP-treated pups was additionally confirmed by the opening of the eyes and ears as well as by the incisor eruption taking place at same age. These results argue for normal physical development of rat pups during the first 2 postnatal weeks after SAP-induced cholinergic impairment. Remarkably, higher and repeated doses of the toxin have been reported to dramatically affect the physical development of the pups, especially their feeding behavior (Robertson et al., 1998).

At the end of the first postnatal week, both control and SAPtreated pups expressed discontinuous oscillatory activity as reported previously for nontreated pups. However, SAP-induced impairment of cholinergic neurons in all BFn differentially affected the properties of SB and NG in the Cg and PL. Although the occurrence of cingulate SB and NG was similar in control and SAP-treated pups, their intrinsic properties were substantially affected by the lesion (Table 1). The significant decrease in the amplitude of SB and NG from $132.02 \pm 9.93$ and 202.74 \pm 10.89 $\mu \mathrm{V}$ to $106.66 \pm 6.99$ and $156.03 \pm 18.74 \mu \mathrm{V}$, respectively (Fig. 3 Eii), was accompanied by a shortening of their duration from $2.74 \pm 0.15$ and $2.99 \pm 0.26 \mathrm{~s}$ to $2.28 \pm 0.12$ and $1.68 \pm 0.23 \mathrm{~s}$, respectively, indicating that the reduced cholinergic input led to a subtle diminishment of the network activity in the Cg. Additionally, the early cholinergic impairment had prominent consequences on the discontinuous activity of the PL. The slight decrease in the duration of prelimbic SB was accompanied by a significant $(p=0.011)$ reduction of the NG occurrence from $0.44 \pm 0.08$ to $0.13 \pm 0.06$ bursts/min ( $n=15$ pups; Fig. 3Ei,Eii) and duration. Remarkably, the maximum amplitude of the remaining NG significantly ( $p=0.008$ ) increased from $122.28 \pm$ 13.77 to $216.9 \pm 17.54 \mu \mathrm{V}$ after SAP injection (Fig. 3Eii). Despite constant dominant frequency within theta range, SAP treatment caused a shift in the relative power of the NG from slow theta to fast beta frequencies. Together, these data indicate that early global depletion of the cholinergic input has the strongest impact on the prelimbic NG by affecting the entrainment of neonatal networks in gamma episodes.

With ongoing maturation, the progressively increasing lesion of cholinergic neurons significantly affected the prefrontal oscillatory activity that switched at approximately P10 from discontinuous events to continuous theta-gamma rhythms (Fig. $3 F$ ). As for the discontinuous patterns, the SAP effects on the cingulate continuous activity were less prominent than on the prelimbic rhythms (Table 1). The prelimbic activity of SAP-treated pups had a significantly $(p=0.001)$ higher amplitude of $306.2 \pm 8.36$ $\mu \mathrm{V}(n=4$ pups $)$ than the control pups $(269.87 \pm 6.31 \mu \mathrm{V}, n=4$ pups). Although the main frequency of continuous thetagamma rhythm did not change as a consequence of SAP treatment, the relative power within the gamma frequency band decreased from $10.8 \pm 0.58$ to $7.81 \pm 0.29 \%$ and within slowfrequency bands increased from $21.3 \pm 0.4$ to $22.59 \pm 0.38 \%$ after the reduction of the cholinergic tone.

Thus, these results demonstrate that early global diminishment of the cholinergic input hampers the local network coupling in gamma episodes and modifies the properties of the underlying slow bursts.

Selective immunotoxic lesion of cholinergic neurons in the MS modifies the prefrontal patterns of oscillatory activity SAP lesion of all cholinergic nuclei has global effects on both neocortical and hippocampal networks, because the $\mathrm{nB}$ mainly 
A

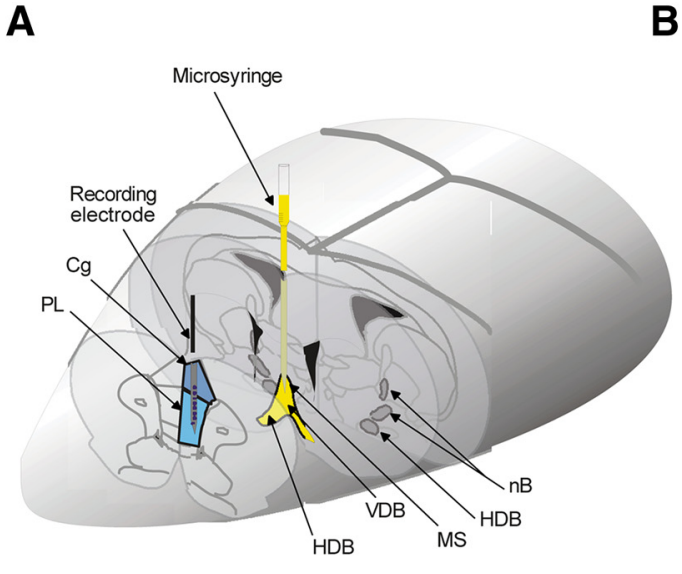

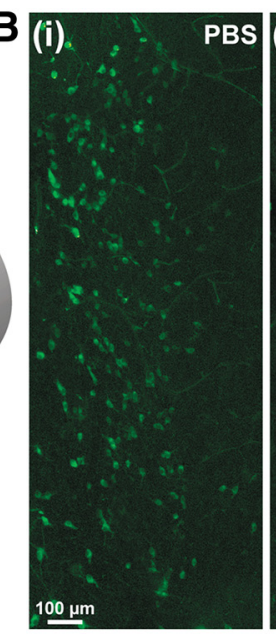

E

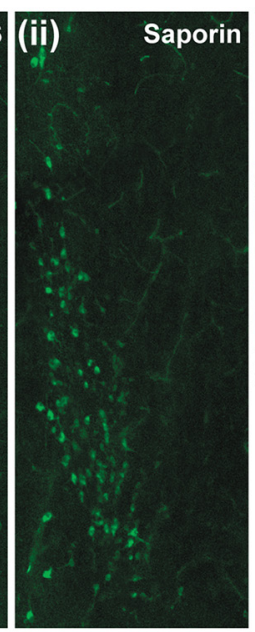

F

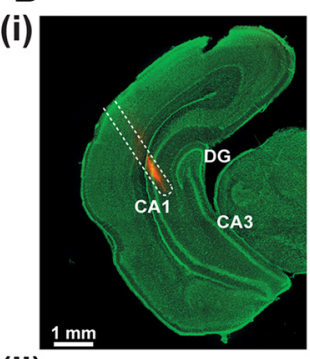

(ii)

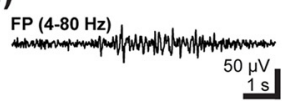

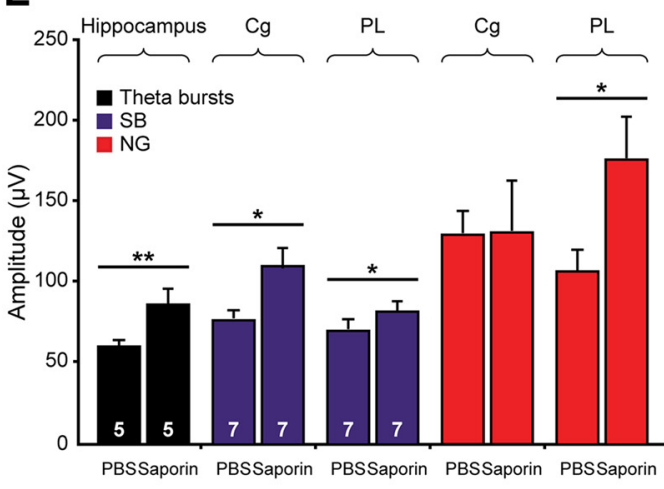

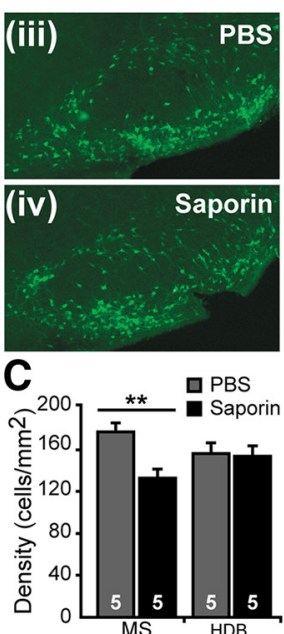

PBS

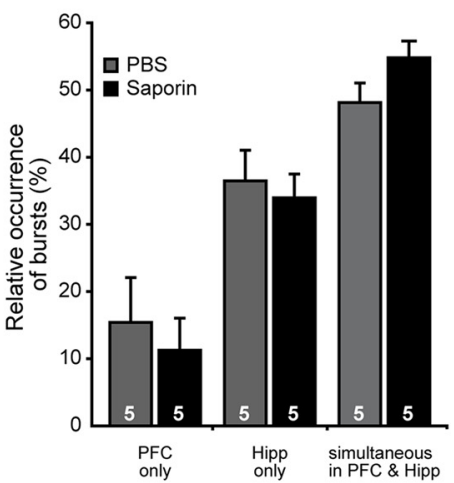

Figure 4. Consequences of selective immunotoxic lesion of the cholinergic MS on the activity patterns within the prefrontal-hippocampal network. $A$, Scheme of experimental setup for recording the PFC (blue) and impairing some cholinergic nuclei (yellow). The spared cholinergic nuclei are marked in dark gray. $\boldsymbol{B}$, Immunohistochemistry of ChAT-positive neurons in the MS (i, ii) and in the HDB (iii, iv) of PBS-treated $(\boldsymbol{i}, \mathbf{i i i})$, and SAP-treated $(\boldsymbol{i i}, \boldsymbol{i v})$ pups. The pups were injected at P0 and investigated at P7. Note the decreased number of cholinergic neurons in the MS but not HDB of SAP-treated pups when compared with PBS-treated rats. C, Bar diagram displaying the density of ChAT-positive neurons in the MS and HDB of five PBS-treated (gray) and five SAP-lesioned (black) pups. Di, Digital photomontage reconstructing the location of the Dil-covered recording electrode (orange) in the intermediate Hipp of a Nissl-stained 50 - $\mu \mathrm{m}$-thick coronal section (green) from a P7 rat. Dii, Characteristic theta burst recorded in the CA1 area of the intermediate Hipp of a P7 rat and displayed after bandpass filtering (4-80 Hz). $E$, Consequences of SAP treatment on the prefrontal and hippocampal patterns of neonatal activity. Bar diagram displaying the mean amplitude of hippocampal theta bursts as well as of SB (blue) and NG (red) in the C $\mathrm{g}$ and PL of PBS- and SAP-treated pups. The white numbers on the bars correspond to the number of investigated pups. $\boldsymbol{F}$, Effects of SAP treatment on the temporal coupling of hippocampal and prefrontal activity. Bar diagram displaying the relative occurrence of oscillatory events that are present either exclusively in the PFC or in the Hipp or simultaneously in both regions of PBS-treated (gray) and SAP-treated (black) pups.

innervates the neocortical areas, whereas the MS and VDB provide the cholinergic innervation of the Hipp (Woolf, 1991). Therefore, the diminishment of prefrontal, especially prelimbic superimposed gamma episodes, after intracerebroventricular SAP treatment might result from decreased cholinergic innervation of the PFC but also from abnormal hippocampal function as a consequence of cholinergic deprivation. We recently showed that the prefrontal and hippocampal oscillations are tightly coupled, with the early discontinuous theta bursts in the Hipp driving the oscillatory activity of the neonatal PFC and especially of the PL (Brockmann et al., 2011). To dissect the mechanisms by which the cholinergic innervation controls the prefrontal activity, we selectively impaired the cholinergic neurons projecting to the Hipp but not to other neocortical areas by injecting SAP in the MS/VDB at P0 (Fig. 4A) and recording simultaneously the PFC and Hipp 7-8 d later. ChAT staining revealed that SAP significantly $(p=0.001)$ decreased the density of cholinergic neurons in the MS from $176.41 \pm 8.88$ cells $/ \mathrm{mm}^{2}$ in PBS-treated pups $(n=5)$ to $133.32 \pm 7.88$ cells $/ \mathrm{mm}^{2}(n=5)$ (Fig. 4 Bi,Bii,C). In contrast, SAP had no effect on the neighboring HDB, in which the number of cholinergic cells remained constant (PBS,
$158.24 \pm 8.44$ cells $/ \mathrm{mm}^{2}$; SAP, $153.43 \pm 10.03$ cells $\left./ \mathrm{mm}^{2}\right)($ Fig. 4 Biii,Biv, C).

SAP treatment affects the theta bursts that represent the dominant pattern of network activity recorded in the CA1 area of the neonatal Hipp (Fig. 4D). Reduction of the cholinergic input from the MS/VDB to the Hipp significantly $(p=0.008)$ augmented the amplitude of theta bursts from $61.03 \pm 3.12$ to $87.16 \pm 8.77 \mu \mathrm{V}(n=$ 5 pups) (Fig. $4 E$ ), whereas their occurrence, dominant frequency, and the relative power were not affected (Table 2). This effect contrasts to the previously described SAP-induced reduction in the amplitude of adult theta activity (Lee et al., 1994; Yoder and Pang, 2005). The different cellular mechanisms underlying the generation of neonatal theta bursts versus adult theta rhythms may account for this discrepancy. Whereas adult theta activity emerges from the interplay between excitatory and inhibitory drives (Lee et al., 1994; Hasselmo, 2005), the neonatal theta bursts may be intrinsically generated (Karlsson and Blumberg, 2003) and involve subcortical triggers in the absence of "classical" GABAergic inhibition (Ben-Ari, 2002). Modification of the hippocampal activity after the diminishment of cholinergic input to the Hipp affected the prefrontal SB and NG differently as the global SAP lesion of all cholinergic nuclei. The 
Table 2. Properties of SB and NG recorded in the $\mathrm{C} g$ and PL and of the hippocampal theta bursts after injection of either PBS or SAP into the MS of neonatal rats

\begin{tabular}{|c|c|c|c|c|c|c|c|c|c|c|}
\hline \multirow[b]{4}{*}{ Properties } & \multicolumn{8}{|c|}{ Discontinuous activity (P7-P8) } & & \\
\hline & \multicolumn{4}{|l|}{$\mathrm{Cg}$} & \multicolumn{4}{|l|}{ PL } & \multirow{2}{*}{\multicolumn{2}{|c|}{$\begin{array}{l}\text { Hippocampal activity (P7-P8) } \\
\text { Theta bursts }\end{array}$}} \\
\hline & \multicolumn{2}{|l|}{ SB } & \multicolumn{2}{|l|}{ NG } & \multicolumn{2}{|l|}{ SB } & \multicolumn{2}{|l|}{ NG } & & \\
\hline & PBS & Saporin & PBS & Saporin & PBS & Saporin & PBS & Saporin & PBS & Saporin \\
\hline Occurrence (bursts/min) & $1.69 \pm 0.3$ & $2.34 \pm 0.28$ & $0.26 \pm 0.08$ & $0.21 \pm 0.1$ & $1.79 \pm 0.33$ & $2.27 \pm 0.4$ & $0.54 \pm 0.17$ & $0.53 \pm 0.14$ & $2.06 \pm 0.14$ & $1.56 \pm 0.12$ \\
\hline Duration (s) & $2.94 \pm 0.18$ & $3.21 \pm 0.17$ & $2.49 \pm 0.24$ & $2.47 \pm 0.31$ & $3.24 \pm 0.2$ & $3.46 \pm 0.2$ & $2.55 \pm 0.32$ & $2.7 \pm 0.23$ & $4.24 \pm 0.21$ & $3.76 \pm 0.16$ \\
\hline Amplitude $(\mu \mathrm{V})$ & $76.41 \pm 4.83$ & $\begin{array}{r}111.06 \pm 11.09 \\
\quad(p=0.01)^{*}\end{array}$ & $130.47 \pm 13.27$ & $134.61 \pm 30.44$ & $69.93 \pm 6$ & $\begin{array}{l}82.87 \pm 6.34 \\
\quad(p=0.012)^{*}\end{array}$ & $107.13 \pm 12.82$ & $\begin{array}{r}177.26 \pm 24.39 \\
\quad(p=0.014)^{*}\end{array}$ & $61.03 \pm 3.12$ & $\begin{array}{l}87.16 \pm 8.77 \\
\quad(p=0.008)^{* *}\end{array}$ \\
\hline Main frequency $(\mathrm{Hz})$ & $6.9 \pm 0.23$ & $6.5 \pm 0.21$ & $7.51 \pm 0.68$ & $5.94 \pm 0.27$ & $6.78 \pm 0.28$ & $6.61 \pm 0.24$ & $7.48 \pm 0.72$ & $7.13 \pm 0.47$ & $6.36 \pm 0.31$ & $6.37 \pm 0.27$ \\
\hline Relative power theta (\%) & $38.22 \pm 1.24$ & $41.58 \pm 1.26$ & $40.22 \pm 4.13$ & $47.97 \pm 3.64$ & $37.47 \pm 1.17$ & $36.89 \pm 1.14$ & $36.05 \pm 1.97$ & $34.05 \pm 1.97$ & $40.52 \pm 1.58$ & $42.6 \pm 2.04$ \\
\hline Relative power alpha (\%) & $24.52 \pm 0.95$ & $24.33 \pm 0.85$ & $23.53 \pm 2.15$ & $22.19 \pm 1.83$ & $23.04 \pm 0.79$ & $23.77 \pm 0.81$ & $21.98 \pm 1.57$ & $\begin{array}{l}28.66 \pm 2.15 \\
\quad(p=0.021)^{*}\end{array}$ & $24.74 \pm 1$ & $23.16 \pm 0.81$ \\
\hline Relative power beta (\%) & $22.36 \pm 0.78$ & $21.69 \pm 1.22$ & $24.74 \pm 2.91$ & $17.63 \pm 1.86$ & $22.5 \pm 0.74$ & $23.29 \pm 1$ & $27.45 \pm 2.39$ & $26.44 \pm 1.71$ & $18.95 \pm 0.98$ & $18.86 \pm 1.07$ \\
\hline
\end{tabular}

Values are presented as mean \pm SEM, and significance values $\left({ }^{*} p<0.05,{ }^{* *} p<0.01,{ }^{* * *} p<0.001\right.$, Mann-Whitney-Wilcoxon test) are indicated in parentheses.

Table 3. Properties of SB and NG recorded in the $\mathrm{Cg}$ and PL of neonatal rats under control conditions (intracortical application of ACSF) and after blockade of $\mathrm{mAChRs} \mathrm{with}$ intracortically applied atropine

\begin{tabular}{|c|c|c|c|c|c|c|c|c|}
\hline \multirow{3}{*}{ Properties } & \multicolumn{8}{|c|}{ Discontinuous activity (P7-P8) } \\
\hline & \multicolumn{4}{|l|}{$\mathrm{Cg}$} & \multicolumn{4}{|l|}{ PL } \\
\hline & \multicolumn{2}{|l|}{ SB } & \multicolumn{2}{|l|}{ NG } & \multicolumn{2}{|l|}{ SB } & \multicolumn{2}{|l|}{ NG } \\
\hline Occurrence (bursts/min) & $1.5 \pm 0.42$ & $1.67 \pm 0.3$ & $0.3 \pm 0.08$ & $0.09 \pm 0.06$ & $1.72 \pm 0.18$ & $\begin{array}{c}2 \pm 0.13 \\
(p=0.043)^{*}\end{array}$ & $0.42 \pm 0.08$ & $\begin{array}{l}0.11 \pm 0.04 \\
(p=0.049)^{*}\end{array}$ \\
\hline Amplitude $(\mu \mathrm{V})$ & $112.91 \pm 17.49$ & $139.88 \pm 14.18$ & $134.4 \pm 16.84$ & $188.97 \pm 49.24$ & $149.98 \pm 13.31$ & $145.59 \pm 12.09$ & $196.93 \pm 27.5$ & $249.99 \pm 43.31$ \\
\hline Main frequency $(\mathrm{Hz})$ & $7.4 \pm 0.4$ & $8.82 \pm 0.83$ & $8.46 \pm 0.65$ & $11.78 \pm 1.56$ & $7.08 \pm 0.3$ & $7.05 \pm 0.32$ & $6.55 \pm 0.54$ & $10.2 \pm 2.98$ \\
\hline Relative power theta (\%) & $34.98 \pm 2.37$ & $\begin{array}{c}28.73 \pm 2.51 \\
(p=0.043)^{*}\end{array}$ & $27.65 \pm 2.72$ & $19.74 \pm 6.98$ & $39.32 \pm 1.35$ & $\begin{array}{c}34.63 \pm 1.41 \\
(p=0.016)^{*}\end{array}$ & $39.12 \pm 2.76$ & $32.59 \pm 5.86$ \\
\hline Relative power alpha (\%) & $23.84 \pm 1.43$ & $\begin{array}{l}18.5 \pm 1.31 \\
(p=0.012)^{*}\end{array}$ & $30.93 \pm 2.46$ & $\begin{array}{c}16.16 \pm 2.36 \\
\quad(p=0.018)^{*}\end{array}$ & $22.91 \pm 1.02$ & $22.18 \pm 0.93$ & $18.85 \pm 1.54$ & $18.46 \pm 3.22$ \\
\hline
\end{tabular}

Values are presented as mean \pm SEM, and significance values $\left({ }^{*} p<0.05,{ }^{* *} p<0.01,{ }^{* * *} p<0.001\right.$, Mann-Whitney-Wilcoxon test) are indicated in parentheses.

amplitude, but not the occurrence, of SB in the Cg and of both SB and NG in the PL was significantly increased and most likely mirrors the augmented amplitude of driving theta bursts in the Hipp (Fig. $4 E$, Table 2). Diminishment of the cholinergic innervation did not affect the coupling through synchronization between the PFC and Hipp. Neither the fraction of oscillatory events occurring simultaneously in both areas [ $48.14 \pm 3 \%$ in PBS-treated pups $(n=5), 54.7 \pm$ $2.5 \%$ in SAP-treated pups $(n=5)]$ (Fig. $4 F)$ nor the theta-band coherence between oscillatory events in the PFC and Hipp (0.41 \pm 0.03 in PBS-treated pups, $0.38 \pm 0.02$ in SAP-treated pups) was significantly modified. These results suggest that direct cholinergic projections to prefrontal GABAergic neurons accounts for the activation of NG episodes, whereas cholinergic modulation of prefrontal-hippocampal networks shapes the amplitude of underlying theta oscillations.

mAChRs but not nAChRs mediate the cholinergic modulation of discontinuous patterns of oscillatory activity in the neonatal PFC

Manipulation of the cholinergic input by either electrical stimulation or SAP-induced lesion profoundly modifies the neonatal oscillatory activity in the PFC by modulating the NG. This effect seems to be attributable to the direct action of cholinergic projections targeting prefrontal GABAergic neurons that entrain local networks in gamma oscillations. To get additional insights into the mechanisms of cholinergic modulation, we investigated the effects of locally applied agonists and antagonists of mAChRs and nAChRs on the prefrontal activity in vivo. Cortical application of the selective $\mathrm{mAChR}$ antagonist atropine $(1 \mu \mathrm{l}, 10 \mathrm{mg} / \mathrm{kg}$ body weight, in ACSF) was performed unilaterally in five P7-P8 pups. Although a very small volume was applied and the speed of injection was very low $(\sim 100-250 \mathrm{nl} / \mathrm{min})$, some of the atropineinduced effects could have been caused by pressure- or lesionrelated action. To exclude any side effects, injections using only vehicle ( $1 \mu \mathrm{l}$ of ACSF) were performed before atropine application in all investigated pups. Although very few and inconsistent differences to the non-manipulated conditions were detected, the patterns recorded in ACSF were considered in all experiments as control values to which the antagonist- or agonist-induced effects were compared.

Atropine slightly modified the discontinuous activity patterns of the neonatal $\mathrm{Cg}$ by reducing the duration of NG from $2.4 \pm$ 0.27 to $1.04 \pm 0.3 \mathrm{~s}$ ( $n=5$ pups, $p=0.028)$ and decreasing their relative power in the alpha frequency band but increasing it in the fast beta and gamma frequency bands (Table 3). The occurrence, amplitude, and main frequency of SB and NG were not affected by the blockade of mAChRs (Fig. 5A, Table 3 ). In contrast, atropine strongly modified the PL activity. The occurrence of NG significantly decreased from $0.42 \pm 0.08$ to $0.11 \pm 0.04$ bursts/ $\min (n=5$ pups), whereas the SB occurrence increased from $1.72 \pm 0.18$ to $2 \pm 0.13$ bursts $/ \mathrm{min}(p=0.043)$ (Fig. $5 A)$. mAChR blockade significantly ( $p=0.032$ ) decreased the firing frequency 

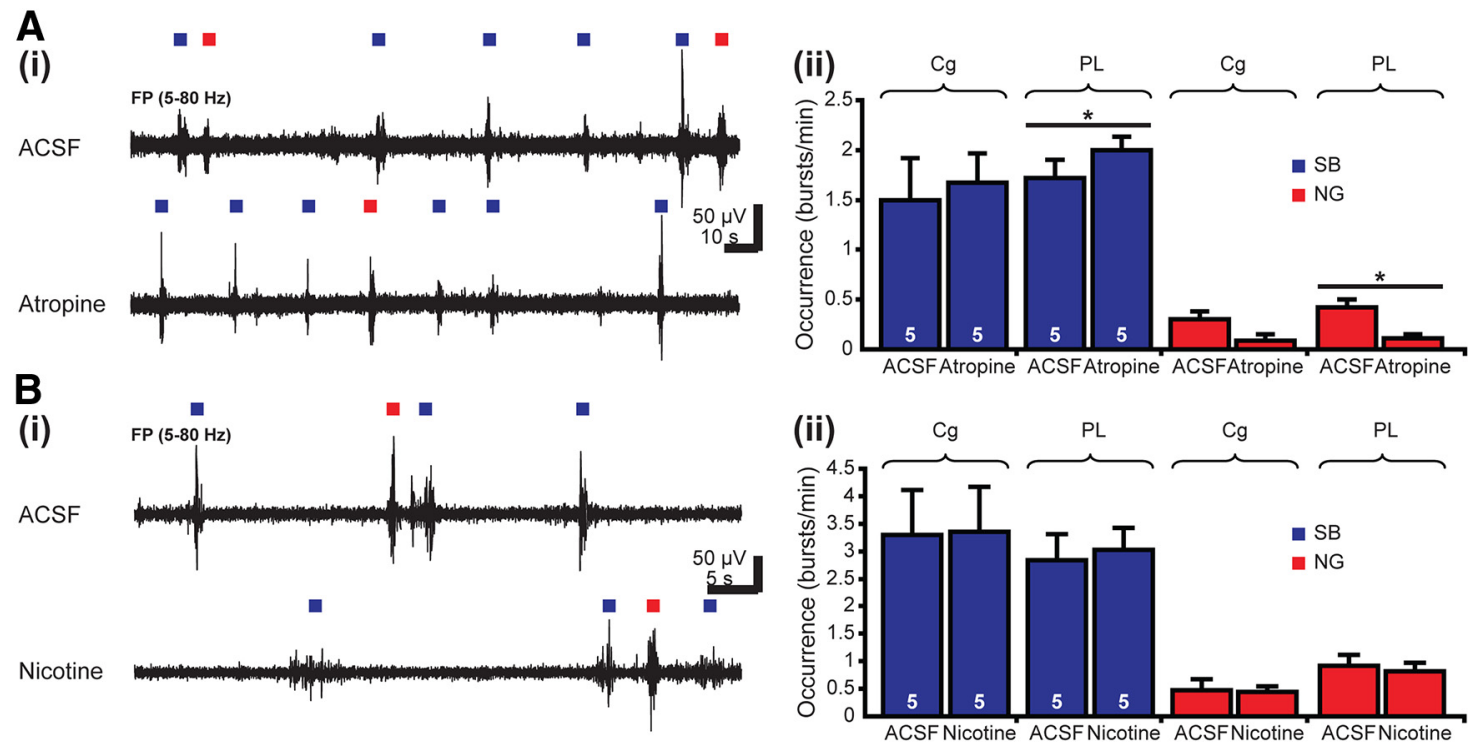

Figure 5. Effects of acute pharmacological manipulations of $m A C h R s$ and $n A C h R s$ on the oscillatory activity of the neonatal PFC. $A$, Consequences of local $m A C h R s$ blockade on SB (blue) and NG (red) in the Cg and PL. Ai, Extracellular FP recordings from the PL of a P7 rat before (ACSF, top trace) and after (bottom trace) intracortical injection of atropine. Blue and red squares differentiate between SB and NG. Aii, Bar diagram displaying the occurrence of SB and NG in the ( $g$ and PL under control conditions (ACSF) and after atropine application in five P7-P8 rats. B, Consequences of local nAChRs activation on SB (blue) and NG (red) in the (g and PL. Bi, Extracellular FP recordings from the PL of a P7 rat before (ACSF, top trace) and after (bottom trace) intracortical injection of nicotine. Bii, Bar diagram displaying the occurrence of SB and NG in the C $g$ and PL under control conditions (ACSF) and after nicotine application in five P7-P8 rats.

of PL neurons from $1.38 \pm 0.24 \mathrm{~Hz}$ under control conditions to $0.82 \pm 0.19 \mathrm{~Hz}$ after atropine application. Additionally, the prelimbic SB were modified after mAChR blockade, with their duration slightly decreasing $(p=0.049)$ from $2.29 \pm 0.15$ to $1.93 \pm 0.16 \mathrm{~s}$ ( $n=5$ pups) (Table 3$)$. These results indicate that $\mathrm{mAChRs}$ mediate the facilitating effect of cholinergic input on the prelimbic gamma episodes.

To decide whether nAChRs equally contribute to the cholinergic modulation of the prefrontal oscillatory activity, we locally applied the specific agonist nicotine $(1 \mu \mathrm{l}, 10 \mathrm{mg} / \mathrm{kg}$ body weight, in ACSF) into the Cg and PL of five P7-P8 pups. Their patterns of oscillatory activity were similar before (in ACSF) and after activation of nAChRs with nicotine (Fig. $5 B$ ), and only the amplitude of NG in the Cg was reduced from $240.75 \pm 30.24$ to $208.39 \pm$ $28.65 \mu \mathrm{V}(p=0.019)$. The absence of consistent nicotine effects was confirmed by the application of the nAChRs antagonist mecamylamine hydrochloride (MLA) (1 $\mu \mathrm{l}, 10 \mathrm{mg} / \mathrm{kg}$ body weight, in ACSF). The SB and NG in the Cg and PL had similar properties under control conditions and after MLA injection, and only the main frequency of NG in the Cg augmented in the presence of MLA from $8.05 \pm 0.72$ to $12.22 \pm 1.38 \mathrm{~Hz}$ ( $n=4$ pups). Neither nicotine nor MLA affected the firing of PL neurons and the generation of gamma episodes. These results indicate that $\mathrm{nAChRs}$ do not mediate the influence of cholinergic input on the PFC.

These findings are surprising, especially in light of the previously reported ability of nAChR-mediated excitability to interfere with the oscillatory activity of the adult neocortex (Armitage et al., 1969; Siok et al., 2006; Lu and Henderson, 2010). One possible explanation for the inability of nAChRs to contribute to the cholinergic control of prefrontal SB and NG might be their functional absence in the neonatal PFC. Previous studies reported that binding sites for the most frequent nAChR subtypes, $\alpha 4 \beta 2$ and $\alpha 7$, are detectable during embryonic development and reached adult features already during the first postnatal week (Tribollet et al., 2004; Huang and Winzer-Serhan, 2006), yet none of them focused specifically on the Cg and PL. Because the specificity of antibodies directed against the epitopes of $\mathrm{nAChR}$

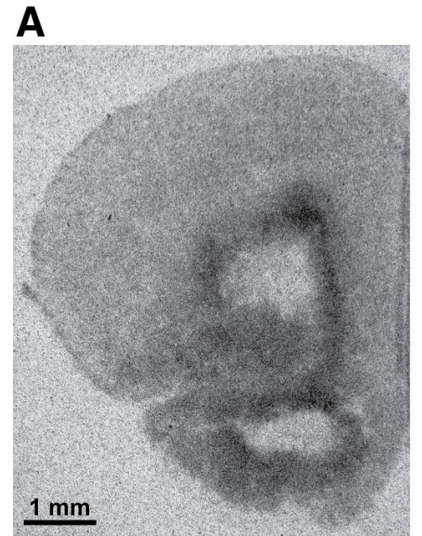

Epibatidine

B

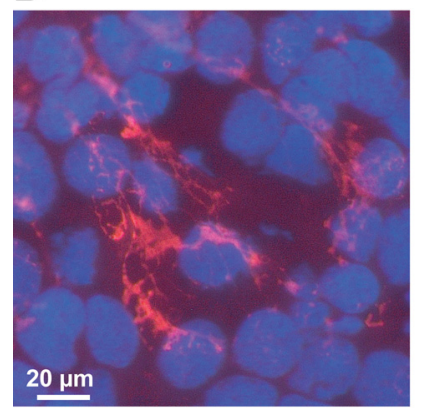

a-bungarotoxin

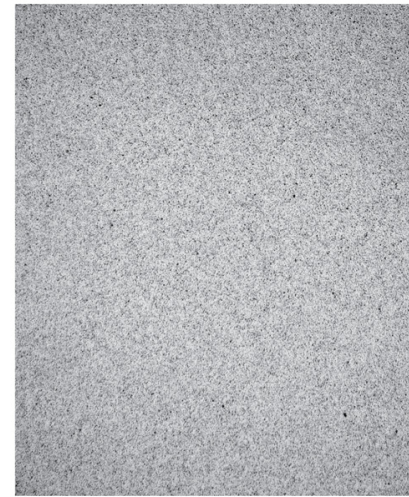

Epibatidine + nicotine

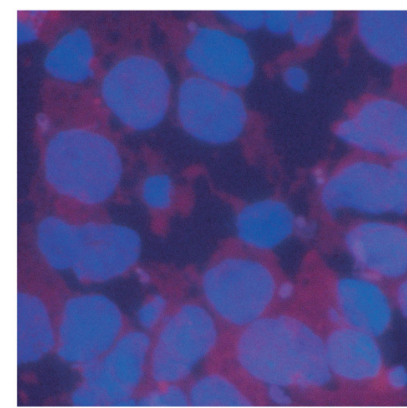

a-bungarotoxin+ nicotine
Figure 6. Presence of functional $\mathrm{mAChRs}$ and $\mathrm{nAChRs}$ in the neonatal PFC of the rat. $\boldsymbol{A}$, Autoradiographs of $\alpha 4 \beta 2 \mathrm{nAChRs}$ using radiolabeled epibatidine obtained from a $20-\mu \mathrm{m}$ thick coronal section of a P6 rat in the presence (right) and absence (left) of nicotine. Note the dense presence of $\alpha 4 \beta 2 \mathrm{nAChRs}$ in the deeper layers of the PFC and the absence of binding when nicotine was added. $\boldsymbol{B}$, Fluorescent staining of $\alpha 7 \mathrm{nAChRs}$ in a $20-\mu \mathrm{m}$-thick coronal section of a P6 pup using Alexa Fluor-555-labeled $\alpha$-bungarotoxin. Note the prominent staining (red) of $\alpha 7 \mathrm{nAChRs}$ in layer II of the PL. 
subunits are still questionable (Moser et al., 2007), we investigated the distribution and density of binding sites for $\alpha 4 \beta 2$ and $\alpha 7$ in the PFC of P6-P7 rats by incubating lightly fixed fresh-frozen coronal sections with either [ $\left.{ }^{125} \mathrm{I}\right]$ epibatidine for targeting $\alpha 4 \beta 2 \mathrm{nAChRs}$ or fluorophore-coupled $\alpha$-bungarotoxin for targeting $\alpha 7 \mathrm{nAChRs}$. $\left[{ }^{125} \mathrm{I}\right]$ Epibatidine binding sites were found widely distributed over the $\mathrm{Cg}$ and PL, whereas preincubation of slices with nicotine led to their disappearance (Fig. 6A). $\left[{ }^{125} \mathrm{I}\right]$ Epibatidine intensely labeled the prelimbic layer VI and the remnants of the subplate and to a lesser extent the layer $\mathrm{V}$. A diffuse labeling of weak intensity was seen through the entire $\mathrm{Cg}$ and the upper layers (II-III) of the PL. $\alpha$-Bungarotoxin binding sites were identified only in the absence of preincubation with nicotine (Fig. 6B) in all layers of the Cg and PL, but the highest density was detected in the upper layers. Thus, both $\alpha 4 \beta 2$ and $\alpha 7$ nAChRs are functionally expressed in the PFC neurons toward the end of the first postnatal week, but they seem to not contribute to the cholinergic control of network activity in the neonatal PFC.

Both mAChRs and nAChRs mediate the cholinergic modulation of continuous patterns of oscillatory activity in the prejuvenile PFC

As shown by the results of SAP lesion, the cholinergic input from the BFn modulates not only the oscillatory activity of the neonatal but also of the prejuvenile $\mathrm{Cg}$ and PL. To decide on the receptor type mediating the cholinergic modulation at this age, we investigated the effects of locally applied agonists and antagonists of mAChRs and nAChRs on the continuous theta-gamma oscillations in the PFC of P14-P15 rats. Blockade of $\mathrm{mAChRs}$ by atropine strongly increased the amplitude of theta rhythm from $146.38 \pm 3.59 \mu \mathrm{V}$ ( $n=5$ pups) in the $\mathrm{Cg}$ and $216.62 \pm 4.23$ $\mu \mathrm{V}$ ( $n=5$ pups) in the PL under control conditions (ACSF) to $348.7 \pm 20.67$ and $262.68 \pm 8.9 \mu \mathrm{V}$, respectively (Fig. $7 A, C$ ). Simultaneously, the oscillatory rhythms became slower as the relative power in the theta frequency band increased and in the beta-gamma frequency band decreased after atropine application (Table 4). Thus, blockade of mAChRs in the prejuvenile PFC had similar effects as reported for the adult neocortex, in which atropine application increased the synchronization of EEG patterns by augmenting their amplitude and power in the $1-5 \mathrm{~Hz}$ range (Metherate et al., 1992). The firing of prefrontal neurons (control, $0.82 \pm 0.11 \mathrm{~Hz}$; atropine, $1.03 \pm 0.11 \mathrm{~Hz} ; n=5$ pups) and the occurrence of gamma episodes superimposed on the theta rhythm (control, $41.11 \pm 0.94$ episodes/min; atropine, $39.74 \pm 1.43$ episodes $/ \mathrm{min} ; n=5$ pups) were not affected by the blockade of mAChRs with atropine. These results suggest that, even if the cholinergic input equally controls the PFC during both neonatal and prejuvenile development, it interacts with differen-
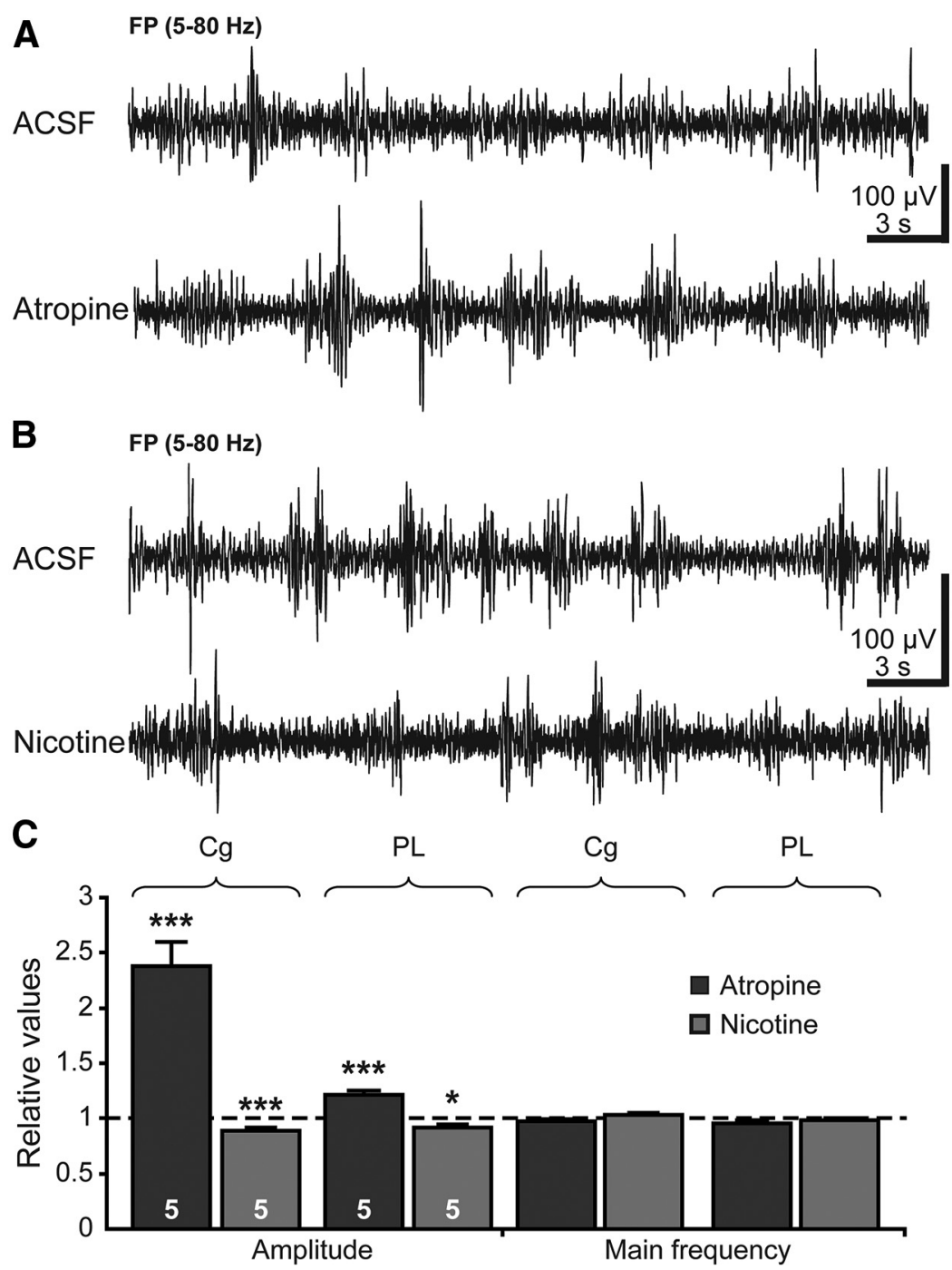

Figure 7. Effects of acute pharmacological manipulations of $\mathrm{mAChRs}$ and $\mathrm{nAChRs}$ on the oscillatory activity of the prejuvenile PFC. $A$, PL of a P14 rat before (ACSF, top trace) and after (bottom trace) intracortical injection of atropine. Note the higher amplitude of theta oscillations in the presence of atropine. $B$, Consequences of local $n A C h R$ activation on continuous theta- gamma oscillations of the $C g$ and Bar diagram displaying the amplitude and main frequency of continuous oscillations in the $\mathrm{G}$ and PL of five atropine-treated (black) and five nicotine-treated (gray) pups normalized to the properties of oscillations after ACSF injection (dotted line).

tially assembled networks during the two stages of development. This hypothesis is supported also by the contribution of nAChRs to the prejuvenile cholinergic control, the manipulation of which changed the continuous theta-gamma rhythms (Fig. 7 B, C). Locally applied nicotine decreased the amplitude of theta rhythms from $229.36 \pm 8.38$ to $204.18 \pm 6.88 \mu \mathrm{V}$ ( $n=5$ pups) in the $\mathrm{Cg}$ and from $244.66 \pm 7.96$ to $225.14 \pm 6.16 \mu \mathrm{V}$ ( $n=5$ pups) in the PL (Table 4). The main frequency remained constant before and after nicotine application, but the relative power shifted from slow theta band to fast beta-gamma band. Similar effects of nicotine on the neocortical EEG patterns have been reported in adult cats and humans (Armitage et al., 1969; Knott, 1988), suggesting that the ability of nAChRs to mediate the cholinergic control of the PFC maturates during the second postnatal week. In contrast, MLA had less prominent effects on the continuous Cg and PL activity (Table 4).

Together, these data indicate that, with ongoing maturation of prefrontal networks and emergence of continuous patterns of 
Table 4. Properties of continuous theta- gamma oscillations recorded in the $\mathrm{Cg}$ and PL of prejuvenile rats under control conditions (intracortical application of ACSF), after blockade of $m A C h R s$ or $n A C h R s$ with intracortically applied atropine or MLA, and after activation of $n A C h R s$ with intracortically applied nicotine

\begin{tabular}{|c|c|c|c|c|c|c|c|c|c|c|c|c|}
\hline \multirow[b]{3}{*}{ Properties } & \multicolumn{12}{|c|}{ Continuous activity (P14-P15) } \\
\hline & \multicolumn{6}{|l|}{$\mathrm{Cg}$} & \multicolumn{6}{|l|}{ PL } \\
\hline & ACSF & Atropine & ACSF & Nicotine & ACSF & MLA & ACSF & Atropine & ACSF & Nicotine & ACSF & MLA \\
\hline Amplitude $(\mu \mathrm{V})$ & $146.38 \pm 3.59$ & $\begin{aligned} 348.7 & \pm 20.67 \\
(p & =4 \mathrm{E}-13)^{* * *}\end{aligned}$ & $229.36 \pm 8.38$ & $\begin{array}{l}204.18 \pm 6.88 \\
\quad(p=2 \mathrm{E}-6)^{* * * *}\end{array}$ & $261 \pm 8.72$ & $247.82 \pm 8.03$ & $216.62 \pm 4.23$ & $\begin{array}{l}262.68 \pm 8.9 \\
\quad(p=2 \mathrm{E}-5)^{* * *}\end{array}$ & $244.66 \pm 7.96$ & $\begin{array}{l}225.14 \pm 6.16 \\
\quad(p=0.036)^{*}\end{array}$ & $277.83 \pm 9.31$ & $\begin{array}{l}251.83 \pm 6.14 \\
\quad(p=0.0004)^{* * * *}\end{array}$ \\
\hline Main frequency $(\mathrm{Hz})$ & $7.02 \pm 0.14$ & $6.84 \pm 0.12$ & $5.74 \pm 0.11$ & $5.92 \pm 0.1$ & $6.17 \pm 0.14$ & $6.02 \pm 0.1$ & $6.55 \pm 0.13$ & $6.32 \pm 0.13$ & $5.97 \pm 0.1$ & $5.88 \pm 0.09$ & $5.95 \pm 0.14$ & $6.06 \pm 0.13$ \\
\hline Relative power theta (\%) & $31.43 \pm 0.55$ & $\begin{aligned} 39.02 & \pm 1.15 \\
(p & =1 \mathrm{E}-18)^{* * *}\end{aligned}$ & $43.55 \pm 0.5$ & $\begin{array}{l}41.11 \pm 0.43 \\
(p=0.004)^{* *}\end{array}$ & $42.49 \pm 0.42$ & $42.89 \pm 0.37$ & $38.67 \pm 0.49$ & $\begin{array}{l}40.61 \pm 0.61 \\
(p=0.001)^{*}\end{array}$ & $44.1 \pm 0.69$ & $\begin{array}{l}40.72 \pm 0.63 \\
(p=7 \mathrm{E}-7)^{* * *}\end{array}$ & $40.43 \pm 0.4$ & $\begin{array}{l}41.6 \pm 0.43 \\
(p=0.011)^{*}\end{array}$ \\
\hline Relative power alpha (\%) & $27.15 \pm 0.4$ & $\begin{aligned} 28.74 & \pm 0.51 \\
(p & =0.015)^{*}\end{aligned}$ & $24.17 \pm 0.25$ & $23.89 \pm 0.3$ & $27.32 \pm 0.36$ & $26.81 \pm 0.34$ & $26.83 \pm 0.32$ & $26.75 \pm 0.39$ & $23.98 \pm 0.26$ & $24.14 \pm 0.29$ & $26.85 \pm 0.42$ & $26.17 \pm 0.41$ \\
\hline Relative power beta (\%) & $23.82 \pm 0.47$ & $\begin{aligned} 19.78 & \pm 0.82 \\
(p & =1 \mathrm{E}-5)^{* * *}\end{aligned}$ & $17.45 \pm 0.42$ & $\begin{array}{l}20.53 \pm 0.54 \\
(p=1 \mathrm{E}-7)^{* * *}\end{array}$ & $15.83 \pm 0.32$ & $16.06 \pm 0.3$ & $19.09 \pm 0.37$ & $18.96 \pm 0.57$ & $18.07 \pm 0.53$ & $\begin{array}{l}21.72 \pm 0.77 \\
(p=3 \mathrm{E}-9)^{* * *}\end{array}$ & $17.2 \pm 0.37$ & $16.92 \pm 0.41$ \\
\hline Relative Power gamma (\%) & $14.31 \pm 0.41$ & $\begin{aligned} 9.25 & \pm 0.64 \\
(p & =2 \mathrm{E}-8)^{* * *}\end{aligned}$ & $5.71 \pm 0.17$ & $\begin{array}{l}6.13 \pm 0.15 \\
(p=0.0002)^{* * *}\end{array}$ & $5.21 \pm 0.17$ & $5.7 \pm 0.21$ & $9.81 \pm 0.32$ & $\begin{array}{l}8.45 \pm 0.36 \\
(p=2 \mathrm{E}-6)^{* * *}\end{array}$ & $5.8 \pm 0.23$ & $\begin{array}{l}6.08 \pm 0.18 \\
(p=0.029)^{*}\end{array}$ & $6.72 \pm 0.26$ & $6.71 \pm 0.29$ \\
\hline Main frequency in gamma band $(\mathrm{Hz})$ & $38.79 \pm 0.56$ & $38.4 \pm 0.44$ & $35.36 \pm 0.4$ & $34.75 \pm 0.31$ & $37.51 \pm 0.47$ & $37.21 \pm 0.41$ & $37.74 \pm 0.53$ & $37.73 \pm 0.44$ & $35.22 \pm 0.4$ & $35.99 \pm 0.46$ & $37.17 \pm 0.42$ & $37.05 \pm 0.34$ \\
\hline
\end{tabular}

Values are presented as mean \pm SEM, and significance values $\left({ }^{*} p<0.05,{ }^{* *} p<0.01,{ }^{* * *} p<0.001\right.$, Mann-Whitney-Wilcoxon test) are indicated in parentheses.

\section{Prefrontal cortex}

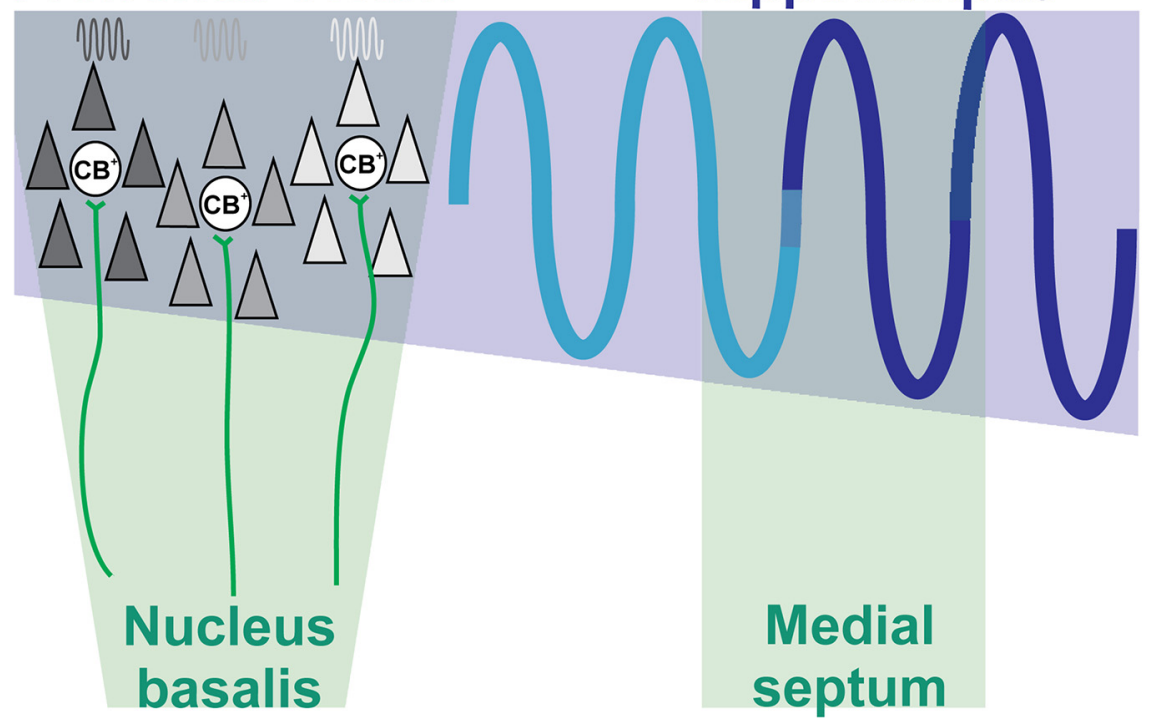

Figure 8. Summary diagram displaying the interactions within the prefrontal-hippocampal network when modulated by subcortical cholinergic nuclei. Cholinergic input (green) from the MS modulates the amplitude of theta drive (blue sine wave) from the Hipp to the PFC. Additionally, cholinergic projections (green) from the nB directly boost the gamma entrainment of local networks in the PFC (dark gray, light gray, white) by acting mainly on CB-positive $\left(C B^{+}\right)$neurons.

activity, both mAChRs and nAChRs mediate the effects of cholinergic input on the prefrontal oscillations.

\section{Discussion}

Cholinergic input from the basal forebrain controls the adult cognitive abilities by modulating the oscillatory entrainment of complex neuronal networks. Here, we investigated the role of ingrowing cholinergic projections for the maturation of activity patterns in the PFC of neonatal and prejuvenile rats and of the oscillatory coupling within developing prefrontal-hippocampal networks. We demonstrate for the first time the following: (1) cholinergic projections reach the PFC toward the end of the first postnatal week and initially target GABAergic neurons; (2) activation of the cholinergic projections boosts the prefrontal oscillatory discharge, especially the prelimbic superimposed gamma episodes of NG; (3) cholinergic innervation modulates the prefrontal activity both directly by facilitating NG and indirectly by controlling the hippocampal theta drive; and (4) mAChRs, but not nAChRs, mediate the neonatal cholinergic modulation, whereas both receptor types act in prejuvenile networks.

\section{Cholinergic modulation of the neonatal PFC}

Several lines of evidence indicate that, during neonatal development, ACh mainly modulates the prelimbic networks locally entrained in short gamma oscillations. In a first instance, repeated electrical stimulation of the BFn at the bursting frequency of cholinergic neurons facilitated the oscillatory entrainment of prelimbic networks by augmenting the occurrence of NG. This effect is attributable to neither stronger cholinergic innervation nor different degrees of functional maturation in the PL versus $C g$. As far as the low number of cholinergic projections initially entering the PFC lets us discriminate, their density or ingrowth dynamics are similar in the neonatal $\mathrm{Cg}$ and PL. Equal distribution of cholinergic projections has been reported also for the adult $\mathrm{Cg}$ and $\mathrm{PL}$ (Mayo et al., 1984; Rye et al., 1984; Gaykema et al., 1990). Moreover, the target of these projections, mAChRs and nAChRs, do not show an area-specific accumulation in the neonatal PFC (Tribollet et al., 2004; present data). Conversely, cholinergic projections to both $\mathrm{Cg}$ and PL are functional toward the end of the first postnatal week, because their activation by single electrical stimuli led with similar efficiency to oscillatory discharge in both areas.

The effects of neonatal cholinergic depletion by SAP represent the second piece of evidence for the preferential cholinergic modulation of prelimbic NG. According to anatomical studies, the lesion of cholinergic BFn starts $24 \mathrm{~h}$ after SAP injection and reaches its maximum 10-17 d later (Robertson et al., 1998; Koh et al., 2005). Despite the fact that $7-8 \mathrm{~d}$ after SAP treatment only $20-50 \%$ of the cholinergic neurons get lost, the occurrence of NG in the PL strongly decreased, whereas their diminishment in the $\mathrm{Cg}$ was not at a significant level. Even if the most prominent and specific SAP effect in the neonatal PFC was the reduction of superimposed gamma episodes, the intrinsic properties of the underlying slow bursts were affected in a similar way as reported 
previously for the neonatal visual cortex (Hanganu et al., 2007). Therefore, the SAP-induced modification of network activity correlates with and might be caused by global diminishment of neocortical activity after cholinergic depletion. This reduced network activity may causally link the morphological abnormalities with the previously identified behavioral consequences of reduced cholinergic drive during development. The SAP-induced reduction of cortical thickness, dendritic branching, and connectivity (Höhmann et al., 1988; Robertson et al., 1998) may destabilize the cortical networks in their ability to sustain oscillatory coupling. In turn, the less frequent and shorter oscillations timing the neurons within local prelimbic networks may worsen the Hebb-like refinement of these networks over the early developmental phase and may contribute to miswiring relevant for mnemonic and executive deficits (Pappas et al., 2005).

The third piece of evidence for the cholinergic control of prelimbic gamma episodes is the result of local pharmacological manipulation of mAChRs and nAChRs in the Cg and PL. Blockade of prefrontal mAChRs decreased the network entrainment in gamma episodes only in the PL but not in the Cg. Remarkably, only mAChRs, but not nAChRs, mediate the cholinergic facilitation on gamma-band activity. These results are in line with previous data that tightly associate the emergence of gamma oscillations with $\mathrm{mAChRs}$ activation in vitro and in vivo (Buhl et al., 1998; Fisahn et al., 1998; Dupont et al., 2006).

All three experimental pieces of evidence indicate that the cholinergic input acting on $\mathrm{mAChRs}$ principally boosts the entrainment of neonatal prelimbic networks in gamma episodes. This specific effect accompanies the overall modulation of theta network oscillations resulting from cholinergic activation of other cortical areas.

\section{Cholinergic modulation of the prejuvenile PFC}

ACh principally modulates the amplitude of continuous oscillatory activity in the prejuvenile PFC by acting on both mAChRs and nAChRs. Both SAP lesion and pharmacological manipulation demonstrated this different effect of cholinergic drive on prejuvenile networks, the magnitude of which does not rely on age-dependent function/expression of mediating receptors. Whether compensatory mechanisms prevent stronger effects of cholinergic depletion remains to be elucidated. Our data demonstrated the presence of functional nAChRs already at the end of the first postnatal week, but they do not contribute to the modulation of network oscillations. Conversely, the full and stable cholinergic depletion induced by SAP (Holley et al., 1994; Leanza et al., 1996) and the similar effects of global SAP-induced cholinergic lesion and local pharmacological blockade indicate that neither an efficiency increase nor side effects attributable to cholinergic deprivation of other cortical areas substantially account for the degree of cholinergic modulation in the prejuvenile PFC. We rather hypothesize that the structure of prefrontal networks entrained in oscillatory rhythms changes with age, and, consequently, the effect of the cholinergic input onto these networks also modifies. Initially, ACh acting on $\mathrm{mAChRs}$ facilitates the firing of neonatal neurons within gamma-coupled local networks by downregulating several slow potassium currents (McCormick, 1993). With ongoing maturation and refinement of connectivity, the prefrontal networks start to be entrained in continuous theta-gamma rhythms. ACh acts on both $\mathrm{mAChRs}$ and $\mathrm{nAChRs}$ and switches the network activity from largeamplitude slow oscillations to low-amplitude fast oscillations by shifting the firing pattern from phasic to tonic discharge (Metherate et al., 1992) and facilitating complex interactions within a large network, including the Hipp and thalamus (Buzsáki et al., 1988).

\section{Cholinergic modulation within the prefrontal-hippocampal network: functional considerations}

The specific cholinergic modulation of prelimbic NG episodes gives new insights into the network dynamics during early development. At adulthood, gamma oscillations, usually associated with the processing of environmental information (Leung, 1998), are present as nested episodes during sleep (Steriade, 2006). Our previous investigation (Brockmann et al., 2011) indicates that similar coupling between slow bursts and fast gamma episodes takes place in the PFC of neonatal rats during urethane anesthesia. These recording conditions mirror the physiological state predominating at this age, because neonatal rats spend most of the time sleeping and urethane optimally mimics all stages of sleep (Bolles and Woods, 1964; Clement et al., 2008). Most likely, the prefrontal gamma episodes emerge in association with the depolarization of locally entrained cortical neurons during "up" states and are the result of both intrinsic cortical circuitry and subcortical activation, as reported previously for adults (Llinás et al., 1991; Sanchez-Vives and McCormick, 2000; Rodriguez et al., 2004; Destexhe et al., 2007; Mena-Segovia et al., 2008). Our data confirm the cholinergic input as an important subcortical activator of prefrontal networks locally entrained in gamma episodes and demonstrate for the first time that this activation is initiated during early neonatal development (Fig. 8). At this age, ingrowing cholinergic projections mainly target prelimbic GABAergic neurons. We hypothesize that these CB-positive neurons act as a "hub" organizing the gamma entrainment of local prelimbic networks, as reported previously for developing Hipp (Bonifazi et al., 2009; Feldt et al., 2011). It remains to be elucidated whether the bursting firing of BFn cholinergic neurons or their coupling in oscillatory rhythms represents the main drive for boosting the activity of these GABAergic neurons and, consequently, the prefrontal network activity. In addition to the cholinergic input, the dense dopaminergic and serotonergic innervation growing into the PFC during the same time window (Schmidt et al., 1982; Berger et al., 1985) may directly or indirectly exert similar prefrontal activation.

The cholinergic input acts on the PFC not only by boosting the gamma entrainment of local networks but also by modulating the prefrontal-hippocampal interactions. The neonatal Hipp receives a strong input from the cholinergic neurons of the MS (Matthews et al., 1974; Aznavour et al., 2005) that, according to our data, modulates the theta bursts in the CA1 by decreasing their amplitude. Because these bursts drive the prelimbic NG, the cholinergic input to Hipp may indirectly control the amplitude of prefrontal theta-alpha-band activity. Thus, ACh modulates distinct properties (occurrence and amplitude) of the prefrontal activity by multiple actions within the prefrontal-hippocampal network (Fig. 8). This double direct and indirect control of the neonatal PFC might ensure a flexible gating of cortical ensembles, facilitating their maturation and later involvement in mnemonic processing during sleep (Wierzynski et al., 2009).

\section{References}

Alleva E, Caprioli A, Laviola G (1989) Litter gender composition affects maternal behavior of the primiparous mouse dam (Mus musculus). J Comp Psychol 103:83-87.

Alonso A, Khateb A, Fort P, Jones BE, Mühlethaler M (1996) Differential oscillatory properties of cholinergic and noncholinergic nucleus basalis neurons in guinea pig brain slice. Eur J Neurosci 8:169-182.

Armitage AK, Hall GH, Sellers CM (1969) Effects of nicotine on electrocortical activity and acetylcholine release from the cat cerebral cortex. Br J Pharmacol 35:152-160.

Arters J, Hohmann CF, Mills J, Olaghere O, Berger-Sweeney J (1998) Sexually di- 
morphic responses to neonatal basal forebrain lesions in mice. I. Behavior and neurochemistry. J Neurobiol 37:582-594.

Ashwell K W, Paxinos G (2008) Atlas of the developing rat nervous system, Ed 3. San Diego: Elsevier Academic.

Aznavour N, Watkins KC, Descarries L (2005) Postnatal development of the cholinergic innervation in the dorsal hippocampus of rat: quantitative light and electron microscopic immunocytochemical study. J Comp Neurol 486:61-75.

Bear MF, Singer W (1986) Modulation of visual cortical plasticity by acetylcholine and noradrenaline. Nature 320:172-176.

Ben-Ari Y (2002) Excitatory actions of GABA during development: the nature of the nurture. Nat Rev Neurosci 3:728-739.

Berger B, Verney C, Febvret A, Vigny A, Helle KB (1985) Postnatal ontogenesis of the dopaminergic innervation in the rat anterior cingulate cortex (area 24). Immunocytochemical and catecholamine fluorescence histochemical analysis. Brain Res 353:31-47.

Bolles RC, Woods PJ (1964) The ontogeny of the behaviour in the albino rat. Anim Behav 12:427-441.

Bonifazi P, Goldin M, Picardo MA, Jorquera I, Cattani A, Bianconi G, Represa A, Ben-Ari Y, Cossart R (2009) GABAergic hub neurons orchestrate synchrony in developing hippocampal networks. Science 326:1419-1424.

Book AA, Wiley RG, Schweitzer JB (1992) Specificity of 192 IgG-saporin for NGF receptor-positive cholinergic basal forebrain neurons in the rat. Brain Res 590:350-355.

Bowen DM, Smith CB, White P, Davison AN (1976) Neurotransmitterrelated enzymes and indices of hypoxia in senile dementia and other abiotrophies. Brain 99:459-496.

Brockmann MD, Pöschel B, Cichon N, Hanganu-Opatz IL (2011) Coupled oscillations mediate directed interactions between prefrontal cortex and hippocampus of the neonatal rat. Neuron 71:332-347.

Buhl EH, Tamás G, Fisahn A (1998) Cholinergic activation and tonic excitation induce persistent gamma oscillations in mouse somatosensory cortex in vitro. J Physiol 513:117-126.

Buzsáki G, Leung LW, Vanderwolf CH (1983) Cellular bases of hippocampal EEG in the behaving rat. Brain Res 287:139-171.

Buzsaki G, Bickford RG, Ponomareff G, Thal LJ, Mandel R, Gage FH (1988) Nucleus basalis and thalamic control of neocortical activity in the freely moving rat. J Neurosci 8:4007-4026.

Clement EA, Richard A, Thwaites M, Ailon J, Peters S, Dickson CT (2008) Cyclic and sleep-like spontaneous alternations of brain state under urethane anaesthesia. PLoS ONE 3:e2004.

Destexhe A, Hughes SW, Rudolph M, Crunelli V (2007) Are corticothalamic "up" states fragments of wakefulness? Trends Neurosci 30:334-342.

Deutsch JA (1971) The cholinergic synapse and the site of memory. Science 174:788-794.

Drachman DA (1977) Memory and cognitive function in man: does the cholinergic system have a specific role? Neurology 27:783-790.

Dupont E, Hanganu IL, Kilb W, Hirsch S, Luhmann HJ (2006) Rapid developmental switch in the mechanisms driving early cortical columnar networks. Nature 439:79-83.

Eckenstein FP, Baughman RW, Quinn J (1988) An anatomical study of cholinergic innervation in rat cerebral cortex. Neuroscience 25:457-474.

Everitt BJ, Robbins TW (1997) Central cholinergic systems and cognition. Annu Rev Psychol 48:649-684.

Feldt S, Bonifazi P, Cossart R (2011) Dissecting functional connectivity of neuronal microcircuits: experimental and theoretical insights. Trends Neurosci 34:225-236.

Fine A, Hoyle C, Maclean CJ, Levatte TL, Baker HF, Ridley RM (1997) Learning impairments following injection of a selective cholinergic immunotoxin, ME20.4 IgG-saporin, into the basal nucleus of Meynert in monkeys. Neuroscience 81:331-343.

Fisahn A, Pike FG, Buhl EH, Paulsen O (1998) Cholinergic induction of network oscillations at $40 \mathrm{~Hz}$ in the hippocampus in vitro. Nature 394:186-189.

Fox WM (1965) Reflex-ontogeny and behavioural development of the mouse. Anim Behav 13:234-241.

Gaykema RP, Luiten PG, Nyakas C, Traber J (1990) Cortical projection patterns of the medial septum-diagonal band complex. J Comp Neurol 293:103-124.

Gil-Bea FJ, Solas M, Mateos L, Winblad B, Ramirez MJ, Cedazo-Minguez A (2010) Cholinergic hypofunction impairs memory acquisition possibly through hippocampal Arc and BDNF downregulation. Hippocampus.
Advance online publication. Retrieved November 1, 2011. doi:10.1002/ hipo.20812.

Goldman-Rakic PS (1995) Cellular basis of working memory. Neuron 14:477-485.

Gu Q (2002) Neuromodulatory transmitter systems in the cortex and their role in cortical plasticity. Neuroscience 111:815-835.

Hahn ME, Lavooy MJ (2005) A review of the methods of studies on infant ultrasound production and maternal retrieval in small rodents. Behav Genet 35:31-52.

Hanganu IL, Luhmann HJ (2004) Functional nicotinic acetylcholine receptors on subplate neurons in neonatal rat somatosensory cortex. J Neurophysiol 92:189-198.

Hanganu IL, Ben-Ari Y, Khazipov R (2006) Retinal waves trigger spindle bursts in the neonatal rat visual cortex. J Neurosci 26:6728-6736.

Hanganu IL, Staiger JF, Ben-Ari Y, Khazipov R (2007) Cholinergic modulation of spindle bursts in the neonatal rat visual cortex in vivo. J Neurosci 27:5694-5705.

Hasselmo ME (2005) What is the function of hippocampal theta rhythm? Linking behavioral data to phasic properties of field potential and unit recording data. Hippocampus 15:936-949.

Hasselmo ME, Bower JM (1992) Cholinergic suppression specific to intrinsic not afferent fiber synapses in rat piriform (olfactory) cortex. J Neurophysiol 67:1222-1229.

Hasselmo ME, Sarter M (2011) Modes and models of forebrain cholinergic neuromodulation of cognition. Neuropsychopharmacology 36:52-73.

Henny P, Jones BE (2008) Projections from basal forebrain to prefrontal cortex comprise cholinergic, GABAergic and glutamatergic inputs to pyramidal cells or interneurons. Eur J Neurosci 27:654-670.

Hohmann CF (2003) A morphogenetic role for acetylcholine in mouse cerebral neocortex. Neurosci Biobehav Rev 27:351-363.

Höhmann CF, Coyle JT (1988) Long-term effects of basal forebrain lesions on cholinergic, noradrenergic and serotonergic markers in mouse neocortex. Brain Res Bull 21:13-20.

Hohmann CF, Brooks AR, Coyle JT (1988) Neonatal lesions of the basal forebrain cholinergic neurons result in abnormal cortical development. Brain Res 470:253-264.

Holley LA, Wiley RG, Lappi DA, Sarter M (1994) Cortical cholinergic deafferentation following the intracortical infusion of 192 IgG-saporin: a quantitative histochemical study. Brain Res 663:277-286.

Huang LZ, Winzer-Serhan UH (2006) Effects of paraformaldehyde fixation on nicotinic acetylcholine receptor binding in adult and developing rat brain sections. J Neurosci Methods 153:312-317.

Jerbi K, Lachaux JP, N'Diaye K, Pantazis D, Leahy RM, Garnero L, Baillet S (2007) Coherent neural representation of hand speed in humans revealed by MEG imaging. Proc Natl Acad Sci U S A 104:7676-7681.

Karlsson KA, Blumberg MS (2003) Hippocampal theta in the newborn rat is revealed under conditions that promote REM sleep. J Neurosci 23:1114-1118.

Khateb A, Mühlethaler M, Alonso A, Serafin M, Mainville L, Jones BE (1992) Cholinergic nucleus basalis neurons display the capacity for rhythmic bursting activity mediated by low-threshold calcium spikes. Neuroscience 51:489-494.

Khazipov R, Sirota A, Leinekugel X, Holmes GL, Ben-Ari Y, Buzsáki G (2004) Early motor activity drives spindle bursts in the developing somatosensory cortex. Nature 432:758 -761.

Knott VJ (1988) Dynamic EEG changes during cigarette smoking. Neuropsychobiology 19:54-60.

Koh S, Santos TC, Cole AJ (2005) Susceptibility to seizure-induced injury and acquired microencephaly following intraventricular injection of saporinconjugated $192 \mathrm{IgG}$ in developing rat brain. Exp Neurol 194:457-466.

Kuczewski N, Aztiria E, Gautam D, Wess J, Domenici L (2005) Acetylcholine modulates cortical synaptic transmission via different muscarinic receptors, as studied with receptor knockout mice. J Physiol 566:907-919.

Leanza G, Nilsson OG, Nikkhah G, Wiley RG, Björklund A (1996) Effects of neonatal lesions of the basal forebrain cholinergic system by $192 \mathrm{immu}$ noglobulin G-saporin: biochemical, behavioural and morphological characterization. Neuroscience 74:119-141.

Lee MG, Chrobak JJ, Sik A, Wiley RG, Buzsáki G (1994) Hippocampal theta activity following selective lesion of the septal cholinergic system. Neuroscience 62:1033-1047.

Leung LS (1998) Generation of theta and gamma rhythms in the hippocampus. Neurosci Biobehav Rev 22:275-290. 
Linke R, Frotscher M (1993) Development of the rat septohippocampal projection: tracing with DiI and electron microscopy of identified growth cones. J Comp Neurol 332:69-88.

Llinás RR, Grace AA, Yarom Y (1991) In vitro neurons in mammalian cortical layer 4 exhibit intrinsic oscillatory activity in the $10-$ to $50-\mathrm{Hz}$ frequency range. Proc Natl Acad Sci U S A 88:897-901.

Lu CB, Henderson Z (2010) Nicotine induction of theta frequency oscillations in rodent hippocampus in vitro. Neuroscience 166:84-93.

Lysakowski A, Wainer BH, Bruce G, Hersh LB (1989) An atlas of the regional and laminar distribution of choline acetyltransferase immunoreactivity in rat cerebral cortex. Neuroscience 28:291-336.

MacIntosh FC, Oboring PE (1955) Release of acetylcholine from the intact cerebral cortex, 19th International Physiological Congress, Montreal, August.

Manns ID, Alonso A, Jones BE (2000) Discharge properties of juxtacellularly labeled and immunohistochemically identified cholinergic basal forebrain neurons recorded in association with the electroencephalogram in anesthetized rats. J Neurosci 20:1505-1518.

Matthews DA, Nadler JV, Lynch GS, Cotman CW (1974) Development of cholinergic innervation in the hippocampal formation of the rat. I. Histochemical demonstration of acetylcholinesterase activity. Dev Biol 36:130-141.

Mayo W, Dubois B, Ploska A, Javoy-Agid F, Agid Y, Le Moal M, Simon H (1984) Cortical cholinergic projections from the basal forebrain of the rat, with special reference to the prefrontal cortex innervation. Neurosci Lett 47:149-154.

McCormick DA (1993) Actions of acetylcholine in the cerebral cortex and thalamus and implications for function. Prog Brain Res 98:303-308.

Mechawar N, Cozzari C, Descarries L (2000) Cholinergic innervation in adult rat cerebral cortex: a quantitative immunocytochemical description. J Comp Neurol 428:305-318.

Mechawar N, Watkins KC, Descarries L (2002) Ultrastructural features of the acetylcholine innervation in the developing parietal cortex of rat. J Comp Neurol 443:250-258.

Mena-Segovia J, Sims HM, Magill PJ, Bolam JP (2008) Cholinergic brainstem neurons modulate cortical gamma activity during slow oscillations. J Physiol 586:2947-2960.

Metherate R, Ashe JH (1991) Basal forebrain stimulation modifies auditory cortex responsiveness by an action at muscarinic receptors. Brain Res 559:163-167.

Metherate R, Cox CL, Ashe JH (1992) Cellular bases of neocortical activation: modulation of neural oscillations by the nucleus basalis and endogenous acetylcholine. J Neurosci 12:4701-4711.

Miranda MI, Bermúdez-Rattoni F (1999) Reversible inactivation of the nucleus basalis magnocellularis induces disruption of cortical acetylcholine release and acquisition, but not retrieval, of aversive memories. Proc Natl Acad Sci U S A 96:6478-6482.

Moser N, Mechawar N, Jones I, Gochberg-Sarver A, Orr-Urtreger A, Plomann M, Salas R, Molles B, Marubio L, Roth U, Maskos U, WinzerSerhan U, Bourgeois JP, Le Sourd AM, De Biasi M, Schröder H, Lindstrom J, Maelicke A, Changeux JP, Wevers A (2007) Evaluating the suitability of nicotinic acetylcholine receptor antibodies for standard immunodetection procedures. J Neurochem 102:479-492.

Muñoz-Cueto JA, Garcia-Segura LM, Ruiz-Marcos A (1991) Regional sex differences in spine density along the apical shaft of visual cortex pyramids during postnatal development. Brain Res 540:41-47.

Pappas BA, Sherren N (2003) Neonatal 192 IgG-saporin lesion of forebrain cholinergic neurons: focus on the life span? Neurosci Biobehav Rev 27:365-376.

Pappas BA, Davidson CM, Fortin T, Nallathamby S, Park GA, Mohr E, Wiley RG (1996) 192 IgG-saporin lesion of basal forebrain cholinergic neurons in neonatal rats. Brain Res Dev Brain Res 96:52-61.

Pappas BA, Payne KB, Fortin T, Sherren N (2005) Neonatal lesion of forebrain cholinergic neurons: further characterization of behavioral effects and permanency. Neuroscience 133:485-492.

Perry DC, Dávila-García MI, Stockmeier CA, Kellar KJ (1999) Increased nicotinic receptors in brains from smokers: membrane binding and autoradiography studies. J Pharmacol Exp Ther 289:1545-1552.

Perry EK, Tomlinson BE, Blessed G, Bergmann K, Gibson PH, Perry RH (1978) Correlation of cholinergic abnormalities with senile plaques and mental test scores in senile dementia. Br Med J 2:1457-1459.

Quinn B, Toga AW, Motamed S, Merlic CA (1995) Fluoro nissl green: a novel fluorescent counterstain for neuroanatomy. Neurosci Lett 184:169-172.
Rasmusson DD, Clow K, Szerb JC (1994) Modification of neocortical acetylcholine release and electroencephalogram desynchronization due to brainstem stimulation by drugs applied to the basal forebrain. Neuroscience 60:665-677.

Robertson RT, Gallardo KA, Claytor KJ, Ha DH, Ku KH, Yu BP, Lauterborn JC, Wiley RG, Yu J, Gall CM, Leslie FM (1998) Neonatal treatment with 192 IgG-saporin produces long-term forebrain cholinergic deficits and reduces dendritic branching and spine density of neocortical pyramidal neurons. Cereb Cortex 8:142-155.

Rodriguez R, Kallenbach U, Singer W, Munk MH (2004) Short- and longterm effects of cholinergic modulation on gamma oscillations and response synchronization in the visual cortex. J Neurosci 24:10369-10378.

Rye DB, Wainer BH, Mesulam MM, Mufson EJ, Saper CB (1984) Cortical projections arising from the basal forebrain: a study of cholinergic and noncholinergic components employing combined retrograde tracing and immunohistochemical localization of choline acetyltransferase. Neuroscience 13:627-643.

Sanchez-Vives MV, McCormick DA (2000) Cellular and network mechanisms of rhythmic recurrent activity in neocortex. Nat Neurosci 3:1027-1034.

Schmidt RH, Björklund A, Lindvall O, Lorén I (1982) Prefrontal cortex: dense dopaminergic input in the newborn rat. Brain Res 281:222-228.

Semba K, Fibiger HC (1989) Organization of central cholinergic systems. Prog Brain Res 79:37-63.

Siapas AG, Wilson MA (1998) Coordinated interactions between hippocampal ripples and cortical spindles during slow-wave sleep. Neuron 21:1123-1128.

Sillito AM, Kemp JA (1983) The influence of GABAergic inhibitory processes on the receptive field structure of $\mathrm{X}$ and $\mathrm{Y}$ cells in cat dorsal lateral geniculate nucleus (dLGN). Brain Res 277:63-77.

Siok CJ, Rogers JA, Kocsis B, Hajós M (2006) Activation of alpha7 acetylcholine receptors augments stimulation-induced hippocampal theta oscillation. Eur J Neurosci 23:570-574.

Sirota A, Montgomery S, Fujisawa S, Isomura Y, Zugaro M, Buzsáki G (2008) Entrainment of neocortical neurons and gamma oscillations by the hippocampal theta rhythm. Neuron 60:683-697.

Steriade M (2006) Grouping of brain rhythms in corticothalamic systems. Neuroscience 137:1087-1106.

Thierry AM, Gioanni Y, Dégénétais E, Glowinski J (2000) Hippocampoprefrontal cortex pathway: anatomical and electrophysiological characteristics. Hippocampus 10:411-419.

Tribollet E, Bertrand D, Marguerat A, Raggenbass M (2004) Comparative distribution of nicotinic receptor subtypes during development, adulthood and aging: an autoradiographic study in the rat brain. Neuroscience 124:405-420.

Umbriaco D, Watkins KC, Descarries L, Cozzari C, Hartman BK (1994) Ultrastructural and morphometric features of the acetylcholine innervation in adult rat parietal cortex: an electron microscopic study in serial sections. J Comp Neurol 348:351-373.

Van Eden CG, Uylings HB (1985) Cytoarchitectonic development of the prefrontal cortex in the rat. J Comp Neurol 241:253-267.

Vaucher E, Hamel E (1995) Cholinergic basal forebrain neurons project to cortical microvessels in the rat: electron microscopic study with anterogradely transported Phaseolus vulgaris leucoagglutinin and choline acetyltransferase immunocytochemistry. J Neurosci 15:7427-7441.

Warburton EC, Brown MW (2010) Findings from animals concerning when interactions between perirhinal cortex, hippocampus and medial prefrontal cortex are necessary for recognition memory. Neuropsychologia 48:2262-2272.

Wierzynski CM, Lubenov EV, Gu M, Siapas AG (2009) State-dependent spike-timing relationships between hippocampal and prefrontal circuits during sleep. Neuron 61:587-596.

Wiley RG, Oeltmann TN, Lappi DA (1991) Immunolesioning: selective destruction of neurons using immunotoxin to rat NGF receptor. Brain Res 562:149-153.

Woolf NJ (1991) Cholinergic systems in mammalian brain and spinal cord. Prog Neurobiol 37:475-524.

Yang JW, Hanganu-Opatz IL, Sun JJ, Luhmann HJ (2009) Three patterns of oscillatory activity differentially synchronize developing neocortical networks in vivo. J Neurosci 29:9011-9025.

Yoder RM, Pang KC (2005) Involvement of GABAergic and cholinergic medial septal neurons in hippocampal theta rhythm. Hippocampus 15:381-392. 